OPEN ACCESS

Edited by:

Chunhe Li,

Fudan University, China

Reviewed by:

Jie Zheng,

ShanghaiTech University, China

Mohit Kumar Jolly,

Indian Institute of Science, India

*Correspondence:

Parul Maheshwar

ppm5104@psu.edu

Reka Albert

rza1@psu.edu

Specialty section:

This article was submitted to

Systems Biology,

a section of the journal

Frontiers in Physiology

Received: 11 May 2020

Accepted: 09 July 2020

Published: 13 August 2020

Citation:

Maheshwari P, Assmann SM and Albert R (2020) A Guard Cell Abscisic Acid (ABA) Network Model That Captures the Stomatal Resting State.

Front. Physiol. 11:927

doi: 10.3389/fphys.2020.00927

\section{A Guard Cell Abscisic Acid (ABA) Network Model That Captures the Stomatal Resting State}

\author{
Parul Maheshwari ${ }^{1 *}$, Sarah M. Assmann ${ }^{2}$ and Reka Albert1,2* \\ ${ }^{1}$ Department of Physics, Penn State University, University Park, PA, United States, ${ }^{2}$ Department of Biology, Penn State \\ University, University Park, PA, United States
}

Stomatal pores play a central role in the control of carbon assimilation and plant water status. The guard cell pair that borders each pore integrates information from environmental and endogenous signals and accordingly swells or deflates, thereby increasing or decreasing the stomatal aperture. Prior research shows that there is a complex cellular network underlying this process. We have previously constructed a signal transduction network and a Boolean dynamic model describing stomatal closure in response to signals including the plant hormone abscisic acid (ABA), calcium or reactive oxygen species (ROS). Here, we improve the Boolean network model such that it captures the biologically expected response of the guard cell in the absence or following the removal of a closure-inducing signal such as ABA or external $\mathrm{Ca}^{2+}$. The expectation from the biological system is reversibility, i.e., the stomata should reopen after the closing signal is removed. We find that the model's reversibility is obstructed by the previously assumed persistent activity of four nodes. By introducing time-dependent Boolean functions for these nodes, the model recapitulates stomatal reopening following the removal of a signal. The previous version of the model predicts $\sim 20 \%$ closure in the absence of any signal due to uncertainty regarding the initial conditions of multiple network nodes. We systematically test and adjust these initial conditions to find the minimally restrictive combinations that appropriately result in open stomata in the absence of a closure signal. We support these results by an analysis of the successive stabilization of feedback motifs in the network, illuminating the system's dynamic progression toward the open or closed stomata state. This analysis particularly highlights the role of cytosolic calcium oscillations in causing and maintaining stomatal closure. Overall, we illustrate the strength of the Boolean network modeling framework to efficiently capture cellular phenotypes as emergent outcomes of intracellular biological processes.

Keywords: stomatal closure, guard cell, Boolean network, Boolean model, memory, signal transduction

\section{INTRODUCTION}

Stomatal pores on the surfaces of leaves play an important role in allowing uptake of $\mathrm{CO}_{2}$ for photosynthesis and water vapor loss in transpiration. Guard cell pairs surround these stomatal pores and control their aperture by dynamic shrinking and swelling. Guard cells respond to numerous environmental signals, including light of different wavelengths and ambient $\mathrm{CO}_{2}$. In 
response to drought and other desiccating stresses, the plant produces the hormone abscisic acid (ABA), which induces stomatal closure. The process of stomatal closure in response to $\mathrm{ABA}$ involves the activity of many guard cell ion transport proteins, enzymes, and small molecules. There has been significant previous research involving experiments and theoretical and computational analyses to understand this complex process (Li et al., 2006; Ma et al., 2009; Sun et al., 2014).

Given the complex interactions among the many intracellular components of guard cells, network-based dynamic models constitute an efficient method for understanding the system. Dynamic models represent each intracellular component by a qualitative (discrete) or quantitative state variable, which describes the component's abundance or activity. Dynamic models also describe how the interactions and regulatory relationships among components (represented as directed edges in the network) change each component's state variable. Discrete dynamic models are simpler to create than quantitative models (which need extensive parameterization) yet they are rich enough to recapitulate and predict cellular behavior. The simplest type of discrete dynamic model is the Boolean model, wherein each node in the network is assumed to be either in the ON (1) or the OFF (0) state and each node state evolves in time according to its update function. The update function of a node is a logic function of the states of the regulators of the node and is expressed with the AND, OR, and NOT logic operators.

We have previously published network-based discrete dynamic models elucidating the process of stomatal response to various signals, including ABA and light of different wavelengths (Li et al., 2006; Sun et al., 2014; Gan and Albert, 2016; Albert et al., 2017; Maheshwari et al., 2019). These models use extensive iterations between experiments and model simulations. They capture the state corresponding to open or closed stomata as stable final states (attractors) of the dynamical system and they recapitulate almost all experimental results regarding knockout and constitutive activation of various nodes of the network. These models identify important mediators of stomatal closure induced by ABA or by external supply of mediators of ABA-induced closure (such as $\mathrm{Ca}^{2+}$ ). They also identify the various subnetworks that determine the different attractors of the network. These subnetworks, called stable motifs, form generalized positive feedback loops that once stabilized, maintain the constituent nodes in a fixed state (Zañudo and Albert, 2013). The most recent model (Maheshwari et al., 2019) identifies single nodes and combinations of nodes that are sufficient to drive the system to a particular attractor. The model also elucidates various crucial feedback loops that ensure the coordination between different components of the network.

The model of Maheshwari et al. (2019) however, does not recapitulate a few cases where the biological response is open stomata. In the model, stomatal closure is an attractor that is not reversible by the removal of the closure-inducing signal. In contrast, the biological reality in a situation of providing a closure-inducing signal for a limited period is gradual opening of the stomata following removal of the signal (Cummins et al., 1971). Furthermore, this model predicts 20\% closed stomata in the absence of any signal (see Figure 3 of Maheshwari et al., 2019) while the biological reality is that the stomata remain open in the absence of any closure-inducing signal. Here, we present two different modifications of the network model of Maheshwari et al. (2019) such that it recapitulates the expected stomatal behavior in these situations. The first modification corresponds to revising certain assumptions of sustained activities for four nodes in the model. Instead of assuming persistent activity, we incorporate a short-term memory effect for these four nodes, where the node's update function also considers the previous states of its regulator node. To understand the trajectories that lead to the open or closed stomata attractors, we construct the motif succession diagrams for the model version with persistent activity of these nodes as well as for the model version with short-term memory. The second modification corresponds to narrowing down the initial conditions used in the model simulations to ensure that the model recapitulates the open stomata state in the absence of any signal. This analysis elucidates the sensitive dependence of stomatal closure on the initial condition of six nodes whose prestimulus states are currently unknown. We use network analysis and causal logic (Maheshwari and Albert, 2017) to reveal the pathways by which these six nodes can lead to stomatal closure.

\section{MATERIALS AND METHODS}

\section{Background Information on the Boolean Model of ABA-Induced Stomatal Closure}

Multiple iterations of experimental and computational research have led to a successful Boolean network model of ABA induced stomatal closure ( $\mathrm{Li}$ et al., 2006; Albert et al., 2017; Maheshwari et al., 2019). The model relies on extensive literature curation to integrate the signaling components, interactions and mechanisms that underlie ABA-induced stomatal closure. The first version of the network model, containing 40 signaling components (Li et al., 2006), successfully recapitulated knockout phenotypes observed at that time and predicted many new phenotypes. One such prediction, regarding the importance of $\mathrm{pH}$ changes in $\mathrm{ABA}$ induced closure, was experimentally tested and validated in $\mathrm{Li}$ et al. (2006). The model later was expanded to 84 nodes based on new experimental results, for example concerning the identity of ABA receptors (Albert et al., 2017). The model was compared to a full complement of phenotypes observed by wet-bench experimentation and achieved a high degree of agreement. Several predictions of the model with regard to reactive oxygen species, cytosolic $\mathrm{Ca}^{2+}$, and heterotrimeric G-protein signaling were confirmed experimentally in Albert et al. (2017). For ease of simulation and understanding, this larger network model was later reduced to 49 signaling components in a way that preserves the outcomes of the model (Maheshwari et al., 2019).

A significant insight obtained via this model was the recognition of a feedback-rich core that complements a canonical linear ABA signaling pathway. Sustained presence of ABA leads to ABA binding to RCAR/PYR1/PYL receptors (short for soluble pyrabactin resistance 1 /pyrabactin resistance 1 -like regulatory component of $\mathrm{ABA}$ receptor). This leads to the inhibition of the clade-A protein phosphatase 2Cs (PP2Cs), which relieves 
inhibition of the serine-threonine kinase OPEN STOMATA 1 (OST1). Kinase activity of OST1 results in reactive oxygen species (ROS) production, which in turn enhances $\mathrm{Ca}^{2+}$ uptake through the membrane (represented as the node CaIM in the model). This $\mathrm{Ca}^{2+}$ uptake, combined with $\mathrm{Ca}^{2+}$ release from intracellular stores (CIS), leads to cytosolic $\mathrm{Ca}^{2+}$ oscillations, which, together with the sustained presence of $A B A$, lead to production of phosphatidic acid (PA), ROS, and activation of phospholipase $\mathrm{D}$ delta (PLD $\delta$ ). These three components form a positive feedback loop and hence they maintain their activation (Sierla et al., 2016). The cytosolic $\mathrm{Ca}^{2+}$ oscillations directly or indirectly also lead to activation of mitogen-activated protein kinases 9 and 12 (MPK9/12), calcium-dependent protein kinases 3 and 21 (CPK3/21), and depolymerization of microtubules. The ABA signal propagates through these various nodes and feedback loops to ultimately trigger opening of anion channels at the guard cell membrane (Kollist et al., 2014), leading to anion efflux. Anion efflux induces membrane depolarization, which in turn drives $\mathrm{K}^{+}$efflux through depolarization-activated outward $\mathrm{K}^{+}$channels (Ache et al., 2000; Hosy et al., 2003). Solute loss drives the osmotic efflux of water through aquaporins (Grondin et al., 2015), resulting in guard cell deflation and stomatal closure (Roelfsema and Hedrich, 2005). The 84-node network of ABA induced closure can be found as Figure 1 of R. Albert et al. (2017) and the reduced, 49-node version as Figure 1 in Maheshwari et al. (2019). In the version presented here in Figure 1, we incorporate further visual simplifications to ease understanding; we note that our analyses were done on the 49-node network of Maheshwari et al. (2019). The node names used in this paper are the abbreviated node names used in Maheshwari et al. (2019); the full names are indicated in Supplementary Table S1.

Multiple methods of analysis of the Boolean model have been used to understand the dynamics and outcomes of the system under different scenarios. One method is to specify the signal (usually, $\mathrm{ABA}$ ) and the initial states of various components of the network and simulate the trajectory of the system over a given number of time-steps $(\sim 50)$ using the software library BooleanNet (Albert et al., 2008). The initial condition corresponds to the information available on open stomata (see Supplementary Table S2). As there is no biological information available from the experimental literature about the pre-stimulus state of multiple nodes [there are 17 such nodes in the 49node model of Maheshwari et al. (2019)], these nodes were started in a randomly selected initial condition. At each timestep, the nodes of the system are updated (i.e., their states are recalculated) in a randomly selected order (Wang et al., 2012). This is an appropriate update scheme for the stomatal closure network since the timescales of the internal processes are largely unknown. During each simulation, each node in the network changes state one or multiple times and after sufficient time, the system settles down into an attractor. Generally, an attractor can be a fixed point (steady state) or a set of states that repeat indefinitely (a complex attractor, corresponding to a sustained oscillation). The Boolean model of ABA-induced closure yields a complex attractor in which most of the nodes (40 out of 49) have a stationary state. Due to the stochasticity introduced by the update method and by the initial node states, a large number $(\sim 2000)$ of replicate simulations are run. The outcome of the model is summarized as the percentage of simulations in which the node Closure is in the state $1(\mathrm{ON})$ at each time step, which we refer to as the percentage of closure. To characterize a whole time-course of closure in response to $\mathrm{ABA}$ or another signal, the cumulative percentage of closure (CPC) is defined as the sum of the percentage of closure over the course of the simulation (usually 50 time steps). The external supply or constitutive activation of a node is implemented as a sustained ON state, the knockout of a node as a sustained OFF state, and the time-course of a thus-perturbed system is compared to that of the wild-type system (see Section "Simulation of Node Constitutive Activation").

A useful analytical tool for obtaining the system trajectories and attractors of the ABA network is to identify stable motifs, which are generalized positive feedback loops that maintain an associated state of their constituent nodes (see Section "Stable Motifs and Oscillating Motifs" and Zañudo and Albert, 2013). For example, the positive feedback between PA, ROS and PLD $\delta$ determines a stable motif that ensures the sustained ON state of these nodes. Each stable motif can yield the stabilization of further nodes in the network, trapping the system's state into a subset of the state space. One can follow an iterative procedure where the influence of each stable motif is used to reduce the network and one then finds the stable motifs in the reduced network. Some of these secondary stable motifs are dependent on certain conditions such as the prior stabilization of a node. For this reason, they are called conditionally stable motifs (see Section "Stable Motifs and Oscillating Motifs" and Deritei et al., 2019). The ABA-induced closure network also contains a negative feedback loop formed by the nodes $\mathrm{Ca}^{2+}{ }_{c}$ and $\mathrm{Ca}^{2+}$ ATPase (see Figure 1), which under certain conditions (e.g., in the presence of $\mathrm{ABA}$ ) induces the sustained oscillation of these nodes and of a few nodes regulated by them. This negative feedback loop is an example of a conditional oscillating motif (see Section "Stable Motifs and Oscillating Motif").

Starting from a signal and obtaining the consecutive (conditionally) stable motifs and (conditional) oscillating motifs gives us the motif succession diagram, which reflects the system's trajectories and identifies the system's attractors (see Section "Stable Motif Succession Diagrams of the Stomatal Closure Model Versions"). Another complementary approach is to use causal logic analysis to find long-range causal dependencies (i.e., sufficient or necessary subgraphs) or cyclic causal dependencies, which correspond to stable motifs (Maheshwari and Albert, 2017). This method was used to identify the logic backbone of the ABA-induced closure process. For example, $\mathrm{ABA}$ is sufficient for the activation of the node $\mathrm{pH}_{c}$ (meaning an increase in the cytosolic $\mathrm{pH}$ level) and also sufficient for deactivation of the AtRAC1 protein (Maheshwari and Albert, 2017). Causal logic analysis can also be used to find the causal effect of a node perturbation and to identify certain interventions that can independently drive the network into a certain attractor. For example, causal logic indicates that the sustained ON state of the node ROS is a driver of a stable motif corresponding to stomatal 


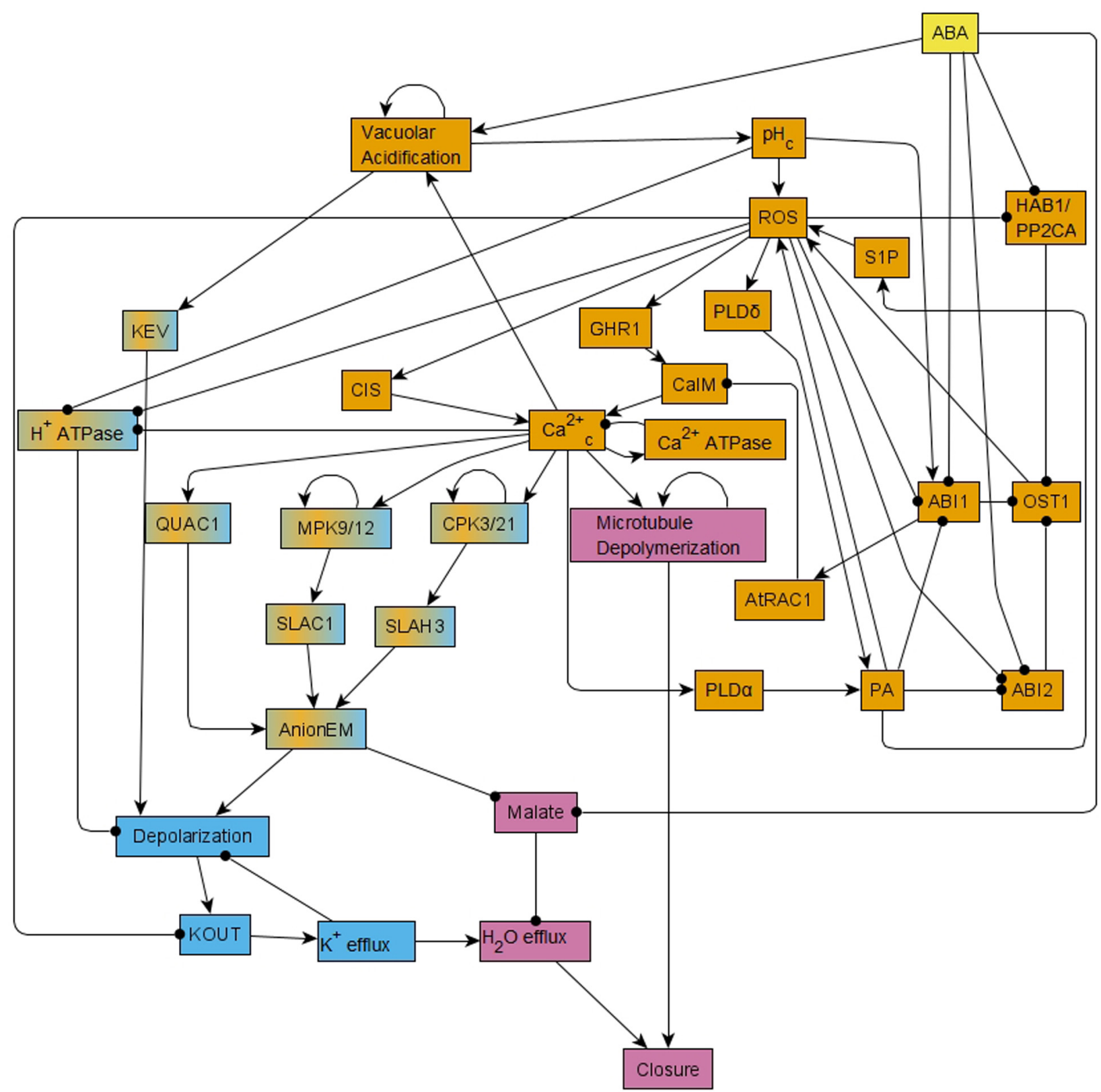

FIGURE 1 | Simplified version of the network that forms the basis of the Boolean model of ABA induced stomatal closure. This network is reduced from the 49-node network in Maheshwari et al. (2019) using methods of causal logic reduction (Maheshwari and Albert, 2017) and binary transitive reduction (Albert et al., 2007); it preserves all the relationships among nodes of the 49-node network via edges or paths. Each edge that terminates in an arrow indicates an activating relationship and each edge that terminates in a black circle indicates an inhibitory relationship. This reduced network is presented here just for ease in visualization; all the analysis in this work was conducted on the 49-node network presented in Figure 1 of Maheshwari et al. (2019). This network contains positive feedback loops, i.e., cycles of directed edges that contain no or an even number of inhibitory edges, for example ROS $-\bullet$ ABI1 $-\bullet$ OST1 $\rightarrow$ ROS. It also contains negative feedback loops, i.e., cycles of directed edges that contain an odd number of inhibitory edges, for example Depolarization $\rightarrow$ KOUT $\rightarrow \mathrm{K}^{+}$efflux $-\bullet$ Depolarization. This network has two strongly connected components (SCCs) i.e., subnetworks in which every pair of nodes is connected by at least two paths of opposite direction. These two SCCs are represented in orange and light blue colors. Nodes of the orange SCC can reach the nodes of the light blue SCC via paths; the nodes linking these two SCCs are shown in a mix of orange and light blue colors. The signal ABA reaches all the nodes of the network. The out-component of this network leads from the two SCCs to the Closure node and is represented in pink color.

closure in the absence of ABA (Maheshwari and Albert, 2017). Indeed, experimental results demonstrate that providing $\mathrm{H}_{2} \mathrm{O}_{2}$ leads to stomatal closure (Zhang et al., 2001; Kwak et al., 2003).
Overall, the results in Albert et al. (2017) illustrate a comprehensive process of model construction from causal relationships, model analysis through simulations and 
network-based methods, model validation, and use of the model to make novel predictions. Most of the discrepancies between model results in Albert et al. (2017) and experimental data involve the model's inability to recapitulate closure in response to the constitutive activation of a node that is causally sufficient for $\mathrm{Ca}^{2+}{ }_{c}$ oscillations. This observation inspired the prediction of a possible $\mathrm{Ca}^{2+}$ regulation of PP2C activity. Following a systematic analysis, Maheshwari et al. (2019) confirm, both in silico and experimentally, that $\mathrm{Ca}^{2+}$ directly or indirectly (via the activation of PA production) inhibits the PP2C ABSCISIC ACID INSENSITIVE 2 (ABI2). The thus-updated model resolves the previous discrepancies. The analysis in Maheshwari et al. (2019) also identifies the stable motif associated with stomatal closure in the absence of ABA, the drivers of this motif, and the causal relationships between various non-canonical closure signals. The basis of our present analysis is the two versions of the model reported in Maheshwari et al. (2019), which we will refer to as Model1 and Model2. The difference between the two models is that in Model1 PA is assumed to inhibit ABI2 while in Model2 $\mathrm{Ca}^{2+}{ }_{c}$ is assumed to inhibit ABI2.

\section{Methods}

\section{Boolean Update Functions}

In a Boolean model the future state of a node is determined by the current state of its upstream regulators and is expressed as a Boolean update function. In the following we will use a simple notation convention for Boolean update functions: represent the state of each node by the name of the node and mark the future state with an asterisk. A node with a single regulator is characterized by one of two types of single variable Boolean functions: identity and negation. Identity is used for positive regulators. Denoting the regulator (and its state) by $\mathrm{X}$ and the target node by $\mathrm{Y}$, the Boolean update function of $\mathrm{Y}$ is $\mathrm{Y}^{*}=\mathrm{X}$, meaning that the target $\mathrm{Y}$ is adopting the state of the regulator $\mathrm{X}$ as its future state. Negation, expressed using the Boolean operator "not," is used for negative regulators. The Boolean update function of $\mathrm{Y}$ is $\mathrm{Y}^{*}=$ not $\mathrm{X}$, meaning that the target $\mathrm{Y}$ adopts the opposite state as the regulator. Notice that in both cases the future state of Y depends only on the state of its regulator $\mathrm{X}$.

For nodes that have more than one regulator, the "or" operator is used if a particular state of any of the regulators can independently activate the target; the "and" operator is used if a particular state of each of the regulators is needed for activation. The choice of the most appropriate operator to use is determined by experimental evidence. Our analysis uses the Boolean update functions constructed in Albert et al. (2017), and then simplified in Maheshwari et al. (2019). Supplementary Table S3 indicates the simplified update functions of each node (as in Maheshwari et al., 2019) and experimental literature supporting it.

\section{Including Memory in Boolean Update Functions}

In most of the Boolean update functions used in models of biological systems the regulators of the target node do not include the node itself, which means that the future state of the node does not depend on its current state. In contrast to differential equation-based models, for which the change in a molecule's concentration is described by explicit synthesis term(s) and degradation term(s) (Tyson and Novak, 2020), the degradation in Boolean models is implicit. For example, the update function $\mathrm{Y}^{*}=\mathrm{X}$ implies that the off state of $\mathrm{X}$ triggers the off state of $\mathrm{Y}$ regardless of whether $\mathrm{Y}$ was on before. In cases where there is a known mechanism for positive self-regulation of a target node and/or there is evidence that the target node's activity is longerlived than the activity of its activators, it is justified to include the target node as its own self-activator. Applying this modification to the previous example yields $\mathrm{X}^{*}=\mathrm{Y}$ or $\mathrm{X}$, which implies that $\mathrm{X}$ will stay in the on state after achieving it for the first time.

There were four such cases in the model of ABAinduced closure (Albert et al., 2017; Maheshwari et al., 2019), namely CPK3/21, MPK9/12, vacuolar acidification and the depolymerization of microtubules. All four of these nodes are activated by an increase in the cytosolic calcium level $\left(\mathrm{Ca}^{2+}{ }_{c}\right)$. However, $\mathrm{Ca}^{2+}{ }_{c}$ elevation cannot be maintained indefinitely because it would lead to precipitation of calcium-phosphates, which would be toxic to the cell. It is also well-documented that $\mathrm{Ca}^{2+}{ }_{c}$ oscillates during the process of stomatal closure (Staxén et al., 1999). Yet, there is no evidence that these four nodes would have an oscillatory pattern, or decrease when the cytosolic $\mathrm{Ca}^{2+}{ }_{c}$ level decreases, during ABA induced stomatal closure. On the contrary, their sustained activation is necessary to achieve sustained anion efflux and stomatal closure. Thus, in Albert et al. (2017) it was assumed that the activity of these four nodes decays very slowly or not at all. This assumption was supported by a specific mechanism in case of three of the four nodes (see Supplementary Text S1). The assumption was implemented by including the state of the node in its own update function, connected by the "or" operator to the state of $\mathrm{Ca}^{2+}{ }_{c}$. For example, the update function of CPK3/21 is CPK3/21 ${ }^{*}=\mathrm{Ca}^{2+}{ }_{c}$ or $\mathrm{CPK} 3 / 21$. According to this function, the next state of CPK3/21 equals $1(\mathrm{ON})$ if $\mathrm{Ca}^{2+}{ }_{c}$ is currently 1 , or if the current state of $\mathrm{CPK} 3 / 21$ is 1 . Once CPK3/21 turns on, it will stay on the whole duration of validity of the model, which represents the 30-60 min needed to achieve closure in response to ABA. We refer to this assumption as the assumed persistent activity of these four nodes.

Here, we study a longer process that consists of stomatal closure induced by $\mathrm{ABA}$, then reopening following the removal of the signal. We therefore introduce an alternative implementation of slow decay of the activity of these four nodes: we include past states of $\mathrm{Ca}^{2+}{ }_{c}$ in their update function. For example, $\mathrm{CPK} 3 / 21^{*}=\mathrm{Ca}^{2+}{ }_{c(t)}$ or $\mathrm{Ca}^{2+}{ }_{c(t-1)}$ or $\mathrm{Ca}^{2+}{ }_{c(t-2)}$ where $\mathrm{t}$ is the current time step. This function indicates that the next state of CPK3/21 equals 1 if $\mathrm{Ca}^{2+}{ }_{c}$ is currently $\mathrm{ON}$ or has been $\mathrm{ON}$ in the last two time steps. We refer to this implementation as shortterm memory. The duration of the short-term memory (in units of a time step) is an adjustable parameter.

\section{Simulation of Node Constitutive Activation}

The constitutive activation (CA) of a node is simulated by setting its state to $\mathrm{ON}$ and keeping it fixed to $\mathrm{ON}$ throughout the entire simulation - this can be thought of as equivalent 
to providing a biomolecule in non-limiting quantity or, for an enzyme, providing the enzyme in non-limiting quantity and in the active state, during an experiment. These in silico mutations are categorized into different response categories (defined in Maheshwari et al., 2019) by their steady state percentage of closure and by their cumulative percentage of closure (CPC) values. In the simulations in the absence of $\mathrm{ABA}$, we refer to the scenario where no node is kept constitutively active as the baseline. Significantly increased response is the case when the percentage of closure reaches $100 \%$. Slightly increased response is the case when the final percentage of closure is higher than the baseline but less than $100 \%$. Close to baseline is the case when the percentage of closure and CPC are within two standard deviations of the respective baseline values. As described in Section "Recapitulating the Open Stomatal State in the Absence of a Signal," baseline percentages of closure can be non-zero.

\section{Evaluation of Consistency Between Simulation and Experiment}

We group the experimental response categories into two broad classes: close to baseline response, which indicates a stomatal aperture that is not statistically significantly different from wild type in the absence of a signal, and increased response (decreased aperture compared to wild type). Slightly or significantly increased response compared to baseline are considered consistent with experimental observation of increased response (decreased aperture compared to the wild type) in the absence of ABA. Close to baseline response is considered consistent with a stomatal aperture measured in the absence of $\mathrm{ABA}$ that is not statistically significantly different from or is greater than that of the wild type.

\section{Stable Motifs and Oscillating Motifs}

A stable motif of a Boolean dynamical system is a generalized feedback loop that maintains an associated state of its constituent nodes regardless of the state of nodes outside of the feedback loop. Stable motifs were first defined using the expanded network, a network that encodes the causal relationships between node states (as incorporated in the update functions). The expanded network consists of two virtual nodes for each node (one for each of the two possible states) and composite nodes that embody AND gates among two or more node states. A stable motif is a subgraph of an expanded network that satisfies four properties: (1) it is strongly connected (there is a path between every pair of nodes in the subgraph), (2) it is consistent (all represented states can be simultaneously satisfied), (3) it is composite-closed (if a composite node is in the subgraph, so too are all its virtual node parents), and (4) it is minimal (it contains no non-trivial subgraphs satisfying the first three properties). Stable motifs can also be defined using the causal logic formalism of Maheshwari and Albert (2017) i.e., a cyclic sufficient subgraph, necessary subgraph, or both sufficient and necessary subgraph determines a stable motif. After the nodes of a stable motif adopt the associated state, they will stay in that state. This may induce other nodes to adopt fixed states as well. Thus, a stable motif determines a region of the state space from which dynamical trajectories cannot escape. In this so-called trap space, a subset of the nodes have a fixed state; for this reason this trap space is also referred to as a partial fixed point.

Another important class of expanded network subgraph is the oscillating motif. Like stable motifs, oscillating motifs are strongly connected, composite-closed subgraphs of the expanded network. Unlike stable motifs, however, oscillating motifs violate the consistency criterion in that every virtual node in the subgraph has its negation in the subgraph as well. Intuitively, oscillating motifs arise from negative feedback loops, while stable motifs arise from positive feedback loops. An oscillating motif will likely (but not always) correspond to a complex attractor in which the nodes represented in the oscillating motif oscillate.

A weaker version of stable motifs was defined by Deritei et al. (2019) and named conditionally stable motif. A conditionally stable motif is a consistent, strongly connected component of the expanded network that is not composite closed. The virtual nodes that are parent nodes of composite nodes included in the conditionally stable motif serve as the conditions. In other words, the conditionally stable motif is a generalized positive feedback loop that maintains an associated state as long as one or more nodes outside of the feedback loop are in a specific state. As long as these parent nodes are in that specific state, the conditionally stable motif behaves like a stable motif. Conditional oscillating motifs can be defined analogously as strongly connected, consistency-violating, not composite-closed subgraphs of the expanded network.

\section{Motif Succession Diagram Analysis}

In general, the stable motif succession diagram is determined by following these steps. First, the system's stable motifs are determined. For every stable motif, the corresponding node states are substituted into the Boolean regulatory functions and the network is iteratively reduced. Then, the stable motifs of the reduced network are determined; these may be stable motifs or conditionally stable motifs of the original network. This process is repeated until the network cannot be reduced anymore. Nodes remaining in the network may oscillate in the attractor(s), while the nodes that were reduced due to the influence of a stable motif will take the associated fixed values. The stable motif succession diagram encapsulates all successions of stable motifs found by this process. For more details, see Zañudo and Albert (2013), where a Java implementation is also provided.

In this paper, we perform motif succession diagram analysis for two assumptions regarding the nodes CPK3/21, MPK9/12, Microtubule Depolymerization and Vacuolar Acidification. The first assumption is persistence of these four nodes, as described in Section "Including Memory in Boolean Update Functions." The second assumption is short term memory for these four nodes; their corresponding motif succession diagrams are detailed in Section "Stable Motif Succession Diagram Analysis of the Network with Short-term Memory." We generate the stable motif succession diagrams for these networks primarily using the Stable Motif code provided in Zañudo and Albert (2013) and supplementing those results by theoretical analysis. The different signal conditions are set by setting the state of the signal node (e.g., ABA or $\mathrm{Ca}^{2+}$ ) to ON or OFF and reducing the network 
with that setting. We augmented these diagrams with our analysis of each conditional oscillatory motif, including identification of its condition and of the nodes whose state stabilizes due to the establishment of this motif. We use the causal logic framework from Maheshwari and Albert (2017) to aid in this analysis.

\section{RESULTS}

\section{Stable Motif Succession Diagrams of the Stomatal Closure Model Versions}

As described in Section "Including Memory in Boolean Update Functions" and Supplementary Text S1, the original model of ABA-induced closure (Albert et al., 2017; Maheshwari et al., 2019) includes the assumption that the activity of four nodes regulated by the cytosolic calcium level $\left(\mathrm{Ca}^{2+}{ }_{c}\right)$, namely CPK3/21, MPK9/12, Vacuolar Acidification and Microtubule Depolymerization, decays very slowly or not at all. The assumption was implemented by introducing their current state in the update function of these nodes. For example, the update function of $\mathrm{CPK} 3 / 21$ is $\mathrm{CPK} 3 / 21^{*}=\mathrm{Ca}^{2+}{ }_{c}$ or $\mathrm{CPK} 3 / 21$. The assumed persistence of the activity of $\mathrm{CPK} 3 / 21$ constitutes a stable motif: once activated, $\mathrm{CPK} 3 / 21$ maintains its $\mathrm{ON}$ state regardless of the rest of the nodes. An analogous stable motif also exists for each of the other three nodes. These stable motifs appear as self-loops on the four nodes in Figure 1. We determined the succession diagrams of stable and oscillating motifs in three cases: absence of ABA, presence of ABA, and externally provided $\mathrm{Ca}^{2+}$. In this section, we present examples of stable motif succession diagrams of Modell and describe the changes observed in Model2.

\section{ABA OFF Case}

As reported in Maheshwari et al. (2019), in the absence of ABA there are 17 attractors. Of these attractors, only one, denoted A0, corresponds to closed stomata. The attractors A1 to A16 are highly similar and are consistent with open stomata (see Supplementary Table S4). These attractors differ in the state of a few nodes that can be stabilized in either the ON or OFF state or can oscillate, while still corresponding to the biologically known information on open stomata.

The network in the ABA $=0$ case has 6 stable motifs, 4 of which correspond to the persistent $\mathrm{ON}$ states of $\mathrm{CPK} 3 / 21$, MPK9/12, Microtubule Depolymerization, and Vacuolar Acidification. The remaining 2 stable motifs, which we denote openM1 and openM2, represent the simultaneous OFF state of more than 10 nodes (see Figures 2A,B).

There also are 5 conditionally stable motifs (CSMs). Four of these represent the persistent OFF state of each of the four self-regulating nodes. The OFF state of CPK3/21, MPK9/12, and Microtubule Depolymerization are conditioned on either openM1 or openM2. The OFF state of Vacuolar Acidification is conditioned on openM1 (it is part of openM2). The fifth CSM, shown in Figure 1C, expresses the self-sustained activity of PLD $\delta, \mathrm{PA}, \mathrm{ROS}, \mathrm{S} 1 \mathrm{P}, \mathrm{OST} 1$, and $\mathrm{pH}_{c}$ together with the sustained inactivity of ABI1 and ABI2. This CSM is dependent on the condition that the Vacuolar Acidification $=1$ stable motif is activated. There are two conditional oscillating motifs. The first is made up by the nodes $\mathrm{Ca}^{2+}{ }_{c}$ and $\mathrm{Ca}^{2+}$ ATPase, both of which oscillate as long as CIS $=1$ or $\mathrm{CaIM}=1$. The second motif comprises the oscillations of the $\mathrm{K}^{+}$efflux, KOUT and Depolarization nodes, conditioned on $\mathrm{KEV}=1$. We refer to this latter motif as $\mathrm{K}^{+}$oscillation. These motifs are presented in Figure 2.

The stabilization of the CSM shown in Figure 1C ensures the establishment of sustained $\mathrm{Ca}^{2+}{ }_{c}$ oscillations. Indeed, if either of the positive regulators of $\mathrm{Ca}^{2+}{ }_{c}$ is stabilized in its active state, the negative feedback between $\mathrm{Ca}^{2+}{ }_{c}$ and $\mathrm{Ca}^{2+}$ ATPase makes both of them oscillate (as characterized in detail in Supplementary Text S2 of Maheshwari et al., 2019). The CSM shown in Figure 1C includes the sustained ON state of the node ROS, which is sufficient for the node CIS. The CSM also includes the sustained OFF state of ABI2, which in combination with the ON state of ROS is sufficient for the node CaIM. Thus, the stabilization of the CSM leads to $\mathrm{Ca}^{2+}{ }_{c}-\mathrm{Ca}^{2+}$ ATPase oscillations. As a result of these oscillations, most nodes directly downstream of $\mathrm{Ca}^{2+}{ }_{c}$, i.e., PLC, PLD $\delta$, QUAC1, TCTP, $\mathrm{V}$-ATPase, DAG, and InsP3/6, also oscillate. Their oscillation periods are given in Supplementary Table S4 and explained in Maheshwari et al. (2019). The remaining two nodes directly regulated by $\mathrm{Ca}^{2+}{ }_{c}$, namely $\mathrm{CPK} 3 / 21$ and $\mathrm{MPK} 9 / 12$, persist in their ON state after first turning on. The oscillations of TCTP stabilize the Microtubule Depolymerization = 1 motif. Altogether, the stabilization of the CSM leads indirectly to the sustained ON state of Microtubule Depolymerization (via sustained $\mathrm{Ca}^{2+}{ }_{c}$ oscillation), and to the sustained $\mathrm{ON}$ state of $\mathrm{H}_{2} \mathrm{O}$ Efflux (via the sustained ON state of multiple nodes downstream of the CSM). The activation of $\mathrm{H}_{2} \mathrm{O}$ Efflux and Microtubule Depolymerization, in turn, leads to stomatal closure. Since this CSM drives the network into an attractor corresponding to closed stomata (see Supplementary Table S4), we will refer to it as closureM.

In conclusion, any system trajectory that (i) involves the stabilization of the stable motifs Vacuolar Acidification $=\mathrm{ON}$, $\mathrm{CPK} 3 / 21=\mathrm{ON}, \mathrm{MPK} 9 / 12=\mathrm{ON}$, Microtubule Depolymerization $=\mathrm{ON}$, closure $M$, and the $\mathrm{Ca}^{2+}{ }_{c}-\mathrm{Ca}^{2+}$ ATPase conditional oscillating motif, and (ii) satisfies the restrictions that Vacuolar Acidification $=\mathrm{ON}$ establishes before closureM and closureM stabilizes before the $\mathrm{Ca}^{2+}{ }_{c}-\mathrm{Ca}^{2+}$ ATPase motif, reaches the closure attractor A0. Figure $3 \mathrm{~A}$ shows two of the possible trajectories to the closed stomata attractor A0 when the first motif to stabilize is Vacuolar Acidification $=\mathrm{ON}$, the second motif to stabilize is MPK9/12 = ON and the third motif to stabilize is $\mathrm{CPK} 3 / 21=\mathrm{ON}$. Figure $\mathbf{3 A}$ also illustrates that if the Vacuolar Acidification stable motif is already locked in then the system's trajectories may bifurcate. In one branch the closure motif stabilizes, leading to the closure attractor A0. In the other branch the openM1 stable motif stabilizes, which leads to the open stomata attractor A1.

Figure 3B denotes two possible motif successions that contain the stable motif openM1 and lead to an attractor that corresponds to open stomata. In both successions, the first motif to stabilize is Microtubule Depolymerization $=\mathrm{ON}$, the second motif to stabilize is MPK9/12 = ON, and the third motif is CPK3/21. openM1 is the condition for the CSMs 


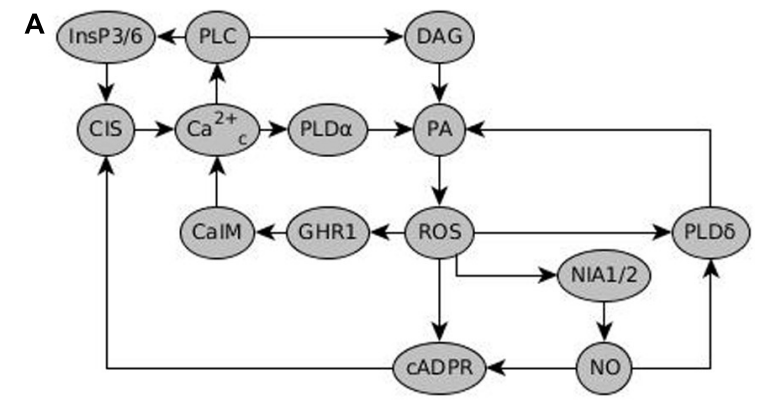

B

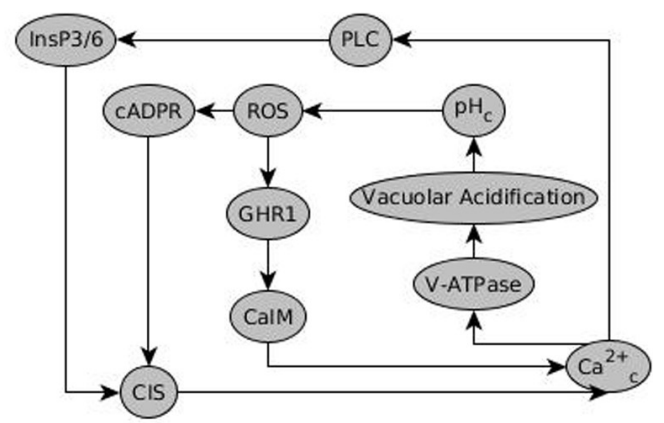

C
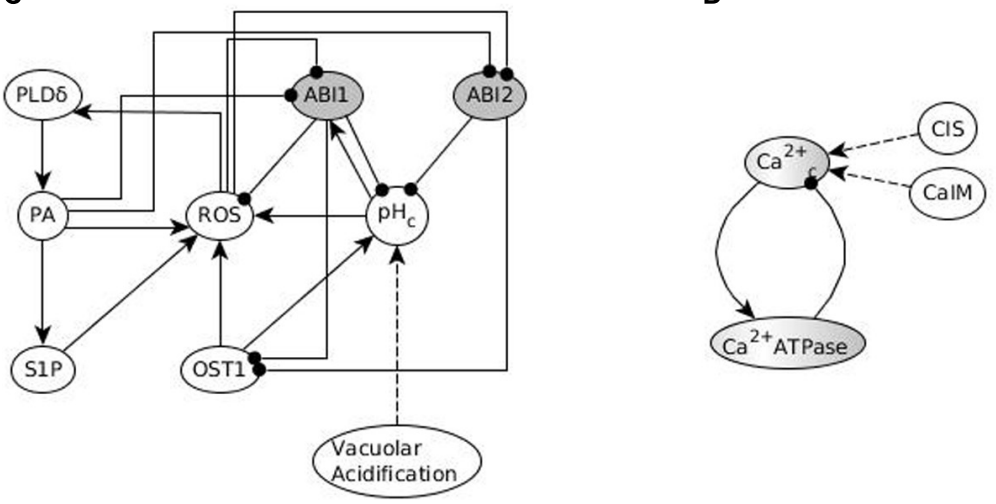

E

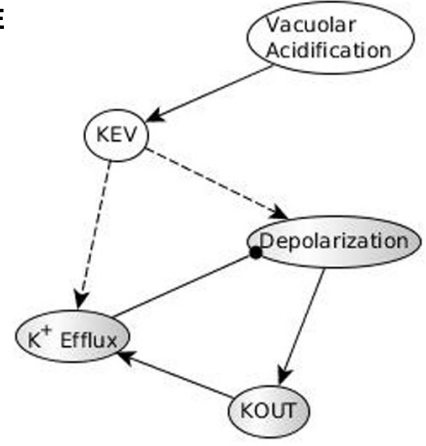

FIGURE 2 Stable and oscillating motifs observed in the absence of ABA. (A) Stable motif associated with open stomata attractors; we refer to this motif as openM1. (B) Stable motif associated with open stomata attractors; we refer to this motif as openM2. (C) Conditionally stable motif associated with the closed stomata attractor A0; we refer to this motif as closureM. The condition for this conditionally stable motif is the ON state of Vacuolar Acidification. In panels (A-C), the white background indicates the ON state of the node and the gray background means OFF state of the node. (D) Conditional oscillatory motif associated with closed stomata. This motif plays a role in both the presence and absence of ABA. The condition for the activation of this motif is the ON state of either of CIS or CalM, and it yields a sustained oscillation of $\mathrm{Ca}^{2+}{ }_{c}$ and $\mathrm{Ca}^{2+}$ ATPase (represented as a gray-white background). (E) Conditional oscillatory motif, $K^{+}$oscillation, that exists in the absence of any signal. The condition for the activation of this motif is KEV = ON. Since Vacuolar Acidification is sufficient for KEV, the ON state of Vacuolar Acidification is sufficient to establish this conditional oscillating motif, leading to sustained oscillation of its constituent nodes.

Vacuolar Acidification = OFF, CPK3/21 = OFF, MPK9/12 = OFF, and Microtubule Depolymerization = OFF. Hence, a possible system trajectory involving openM1 can have both the OFF state and $\mathrm{ON}$ state of the four self-regulating nodes after openM1 is established, while it can have only their ON state before openM1. Any trajectory that contains openM1 and the Vacuolar Acidification $=\mathrm{ON}$ motif leads to the establishment of the conditional oscillating motif $\mathrm{K}^{+}$oscillation. These cases give rise to open stomata attractors that have the $\mathrm{K}^{+}$efflux, KOUT and Depolarization nodes oscillating. Trajectories that contain openM1 and the Vacuolar Acidification = OFF motif lead to open stomata attractors in which $\mathrm{K}^{+}$efflux, KOUT and Depolarization are off. As a result, this case has many possible trajectories and hence leads to a complex succession diagram, one branch of which is displayed in Figure 3B.

Figure 3C denotes a possible succession diagram leading to an open stomata attractor that involve the stabilization of openM2. The Vacuolar Acidification = ON motif is mutually exclusive with openM2. Similar to the case of openM1, the OFF states of CPK3/21, MPK9/12 and Microtubule Depolymerization are CSMs with openM2 as the condition. Hence, a possible system trajectory involving openM2 can have either the ON or OFF state of the three self-regulating nodes after openM2 but it can have only their ON state before openM2. Figure 3C shows the case when the first motif to stabilize is MPK9/12 = ON and the second is CPK3/21.

\section{ABA ON Case}

In the presence of $\mathrm{ABA}$, the network has four stable motifs. Three of these express the self-sustained activity of CPK3/21, MPK9/12, and Microtubule Depolymerization, respectively; these were stable motifs in the absence of ABA as well. The self-loop of Vacuolar Acidification does not appear as a stable motif because the ON state of Vacuolar Acidification is indirectly determined by ABA and does not need self-stabilization. The fourth motif is a cycle that sustains the ON state of PLD $\delta, P A$, and ROS. This cycle is a subset of the closureM motif; the rest of the nodes of this motif are stabilized by ABA. There is also an oscillatory motif comprised of $\mathrm{Ca}^{2+}{ }_{c}$ and $\mathrm{Ca}^{2+}$ ATPase. It is the same as the conditional oscillating motif in the $\mathrm{ABA}=\mathrm{OFF}$ case, however, in the $\mathrm{ABA}=\mathrm{ON}$ case, it is not conditional anymore, because the sustained presence of ABA leads to the ON state 

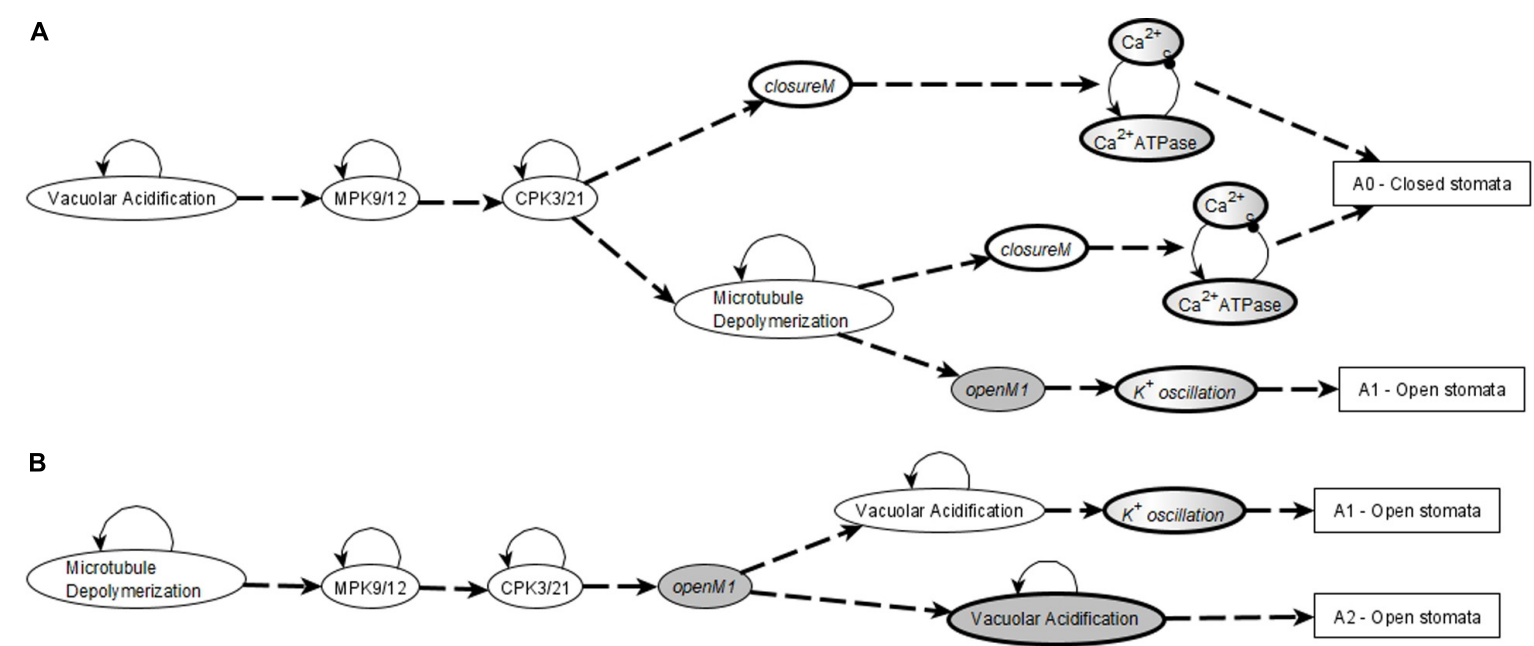

C

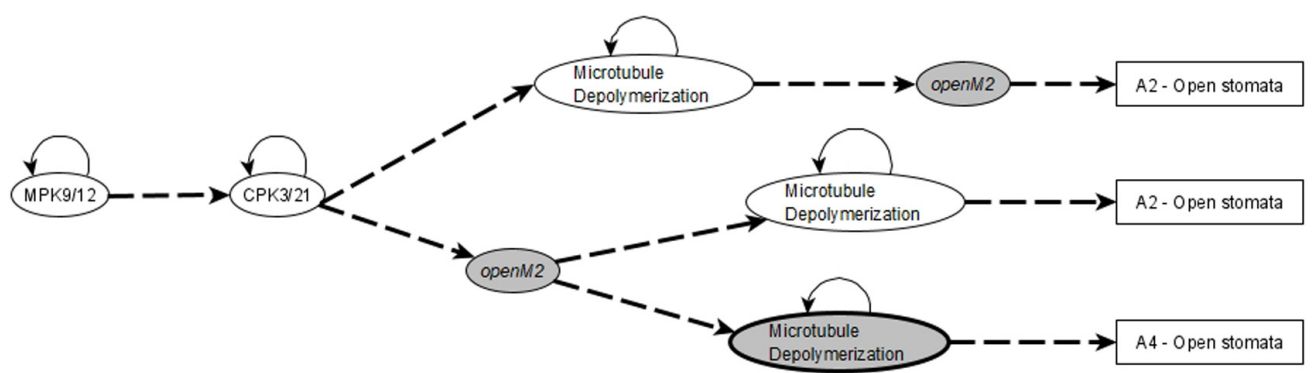

FIGURE 3 | Motif succession diagrams of Model1 in the absence of ABA and any other closure signal. Stable motifs are shown with oval symbols and attractors are indicated by rectangles. Each dashed directed line between two motifs indicates that the system states in which the first motif has established and any nodes driven by it have stabilized admit the second motif as next to activate. The dashed directed line converging into an attractor symbol indicates that the succession of stable motifs ensures the system's convergence into the respective attractor. (A) shows a subset of the succession diagram that converges to the attractor A0, corresponding to closure of the stomata in the absence of ABA (see Supplementary Table S4). The stable motif characteristic to this attractor is the conditionally stable motif closureM. (B,C) show a subset of the succession diagram corresponding to a sample of the 16 attractors that describe open stomata in the absence of ABA (see Supplementary Table S4). Each possible trajectory in this case contains exactly one of the stable motifs openM1 and openM2. (B) describes some of the trajectories containing openM1 while $\mathrm{C}$ describes some of the trajectories containing openM2. (A) also indicates the existence of bifurcations in the system's trajectory due to the mutually exclusive motifs closure $\mathrm{M}$ and openM1. If closureM stabilizes the system converges into the attractor $\mathrm{A} 0$, and if openM1 stabilizes the system converges into attractor A1. The diagram encodes node states into the background color of the stable motif symbols. When referring to single nodes, white background indicates the ON state of the node, gray background means the OFF state of the node, and gray-white background represents oscillating nodes. Since in the openM1 and openM2 stable motifs all nodes are OFF (see Figure 2), we use a gray background color for these stable motifs. We use white background to represent the locking in of the closure $M$ motif and represent the oscillating nature of the $K^{+}$oscillation motif by a gray-white background. The conditionally stable motifs are marked by thick boundaries.

of CaIM, which fulfills the condition for $\mathrm{Ca}^{2+}{ }_{c}-\mathrm{Ca}^{2+}$ ATPase oscillations. The stabilization of these four motifs, in any order, leads to the sole attractor reachable in this case. As expected, and consistent with the knowledge that ABA is sufficient for stomatal closure, this attractor corresponds to closed stomata. It differs from the closure attractor A0 reached in the absence of ABA in the state of five nodes whose state is determined solely by ABA: RCARs, V-PPase, Actin reorganization (which are ON in the presence of ABA and OFF in its absence) and PEPC, AtRAC1 (which are OFF in the presence of $\mathrm{ABA}$ and $\mathrm{ON}$ in its absence). A subset of the motif succession diagram for this case is presented in Figure 4A.

\section{Externally Provided $\mathrm{Ca}^{2+}$ as Signal}

In the absence of $\mathrm{ABA}$, external $\mathrm{Ca}^{2+}$ can be simulated either as the fixed $\mathrm{ON}$ state of the $\mathrm{Ca}^{2+}{ }_{c}$ node or as the fixed ON state of the CaIM node, which represents calcium influx through the membrane. In the case of fixed $\mathrm{ON}$ state of the $\mathrm{Ca}^{2+}{ }_{c}$ node the stable motifs in the network corresponding to closed stomata (i.e., the four self-regulating nodes and the closureM motif) are quickly stabilized, because $\mathrm{Ca}^{2+}{ }_{c}$ is a driver node for all of them. Thus, any initial condition leads to an attractor corresponding to closed stomata. This attractor is slightly different from the attractor corresponding to closure induced by ABA in that there are no oscillating nodes (see Supplementary Table S4). In fact, the effect of fixed $\mathrm{ON}$ state of $\mathrm{Ca}^{2+}{ }_{c}$ is slightly stronger than the effect of the sustained presence of ABA, which leads to sustained $\mathrm{Ca}^{2+}{ }_{c}$ oscillations. This is because the fixed $\mathrm{ON}$ state of $\mathrm{Ca}^{2+}{ }_{c}$ drives the network into a closed stomata attractor in fewer time steps than the sustained presence of ABA.

When the external $\mathrm{Ca}^{2+}$ is simulated as the fixed $\mathrm{ON}$ state of the CaIM node, the network has all of the motifs 


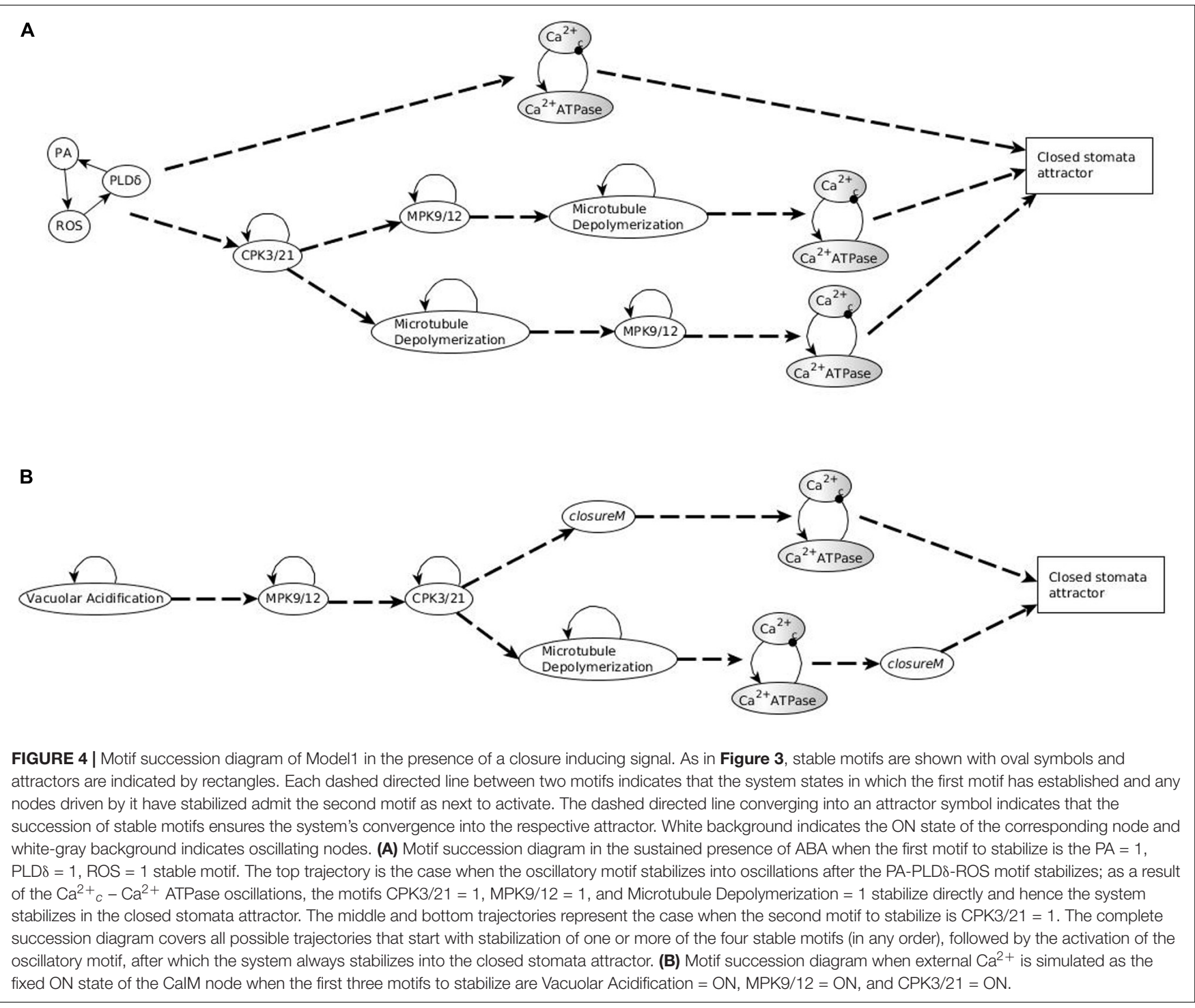

associated with the closed stomata attractor in the absence of $\mathrm{ABA}$, i.e., the four stable motifs corresponding to persistent activity, the ClosureM motif and the oscillatory motif containing $\mathrm{Ca}^{2+}{ }_{c}$ and $\mathrm{Ca}^{2+}$ ATPase (whose condition is now satisfied). The succession diagram of this case, shown in Figure $4 \mathrm{~B}$, is very similar to the one shown in Figure $\mathbf{3 A}$ except that the $\mathrm{Ca}^{2+}{ }_{c}-\mathrm{Ca}^{2+}$ ATPase oscillating motif is not a conditional motif anymore and can hence form a trajectory by appearing in any order with the four stable motifs. The closureM motif is still a conditionally stable motif. Figures $3 \mathrm{~B}, \mathrm{C}$ are not possible in this case since the ON state of CaIM is incompatible with the openM1 and openM2 motifs. In other words, the only attractor possible in this case is the closed stomata attractor A0.

The stable motifs and the succession diagram of Model2, i.e., the network model in which $\mathrm{Ca}^{2+}{ }_{c}$ directly inhibits $\mathrm{ABI}$, are the same as those of Modell in the presence of ABA and in the presence of external calcium. There are some small differences between the models in the absence of ABA, which are described in Supplementary Text S2.

\section{Relaxation to Resting State After Removing the Signal}

According to our analysis of the model in Maheshwari et al. (2019), stomatal closure involves the stabilization of four stable motifs, the ClosureM conditionally stable motif (whose condition is the Vacuolar Acidification stable motif) and a $\mathrm{Ca}^{2+}{ }_{c}$ - $\mathrm{Ca}^{2+}$ ATPase conditional oscillating motif (whose condition is satisfied by the ClosureM motif, see Section "Stable Motif Succession Diagrams of the Stomatal Closure Model Versions"). The four stable motifs stay in their associated state once stabilized. Even if the signal is taken away in the model, these stable motifs still stay stabilized, and so do the conditional motifs (since their condition is still satisfied), keeping the simulated stomata closed. Figure 5 shows a simulation of Model1, 
indicating that removal of the signal (ABA) does not lead to re-opening of the simulated stomata. This is not accurate since the biological reality is the reopening of the stomata after ABA is removed (Cummins et al., 1971). Here, we resolve this discrepancy by making the activity of certain nodes less persistent than assumed in Albert et al. (2017) and Maheshwari et al. (2019).

As described in Section "Including Memory in Boolean Update Functions," the model currently assumes persistent activity of CPK3/21, MPK9/12, Microtubule Depolymerization and Vacuolar Acidification, which form stable motifs (see Figure 3). These stable motifs make the closure attractor irreversible, which is not biologically accurate. Hence, we considered the possibility of decreasing the persistence of these nodes, i.e., we assumed that the state of the target node is sustained only for a few time steps after its regulator (e.g., $\mathrm{Ca}^{2+}{ }_{c}$ ) turns off, after which the target node goes back to the OFF state. We implement this short-term memory by storing the state of the regulator in the past few timesteps (see Figure 6). Specifically, we use auxiliary nodes to remember the past states of the regulator (e.g., $\mathrm{Ca}^{2+}{ }_{c}$ ); these auxiliary nodes are always updated before the rest of the nodes are updated.

We use the ranked asynchronous update of the BooleanNet software library, in which the nodes are classified as rank 1, $2, \ldots$ and during each timestep, nodes of rank 1 are updated first (according to a random order among these nodes), followed by nodes of rank 2, and so on. We designate the auxiliary nodes corresponding to the largest memory as rank 1 , the auxiliary nodes with next largest memory as rank 2, and so on. The regular nodes of the network have the lowest rank (highest numerical value). For example, when Microtubule Depolymerization considers the last five timesteps of TCPT, CPK3/21 and MPK 9/12 consider the last three timesteps of $\mathrm{Ca}^{2+}{ }_{c}$ and Vacuolar Acidification considers the last two timesteps of V-ATPase (as in Figure 7), the auxiliary nodes TCTP_5, Ca_3 and V-ATPAse_2 have rank 1, the auxiliary nodes TCTP_4, Ca_2 and V-ATPase_1 have rank 2, the auxiliary nodes TCTP_3 and Ca_1 have rank 3, the auxiliary node TCTP_4 has rank 4, the auxiliary node TCTP_ 5 has rank 5 and all the regular nodes have rank 6. The Boolean update functions for this case are given in Supplementary Text S3.

We implemented short-term memory effect for the four nodes and explored various durations for each of them to identify cases which show efficient closure in response to $\mathrm{ABA}$ and efficient reopening of the stomata after ABA is removed. The analysis of these durations, described in Supplementary Text S4, indicates that a memory of three timesteps ensures the persistent ON state of CPK3/21 and MPK9/12, while the persistence of Microtubule Depolymerization or Vacuolar Acidification would be ensured for a memory of six timesteps. We found that the best result is exhibited by the model version where Vacuolar Acidification considers the last 2 time-steps of V-ATPase, CPK3/21 and MPK9/12 each consider the last 3 time-steps of $\mathrm{Ca}^{2+}{ }_{c}$, and Microtubule Depolymerization considers the last 5 time-steps of TCTP. Figure 7 shows the re-opening of stomata after the removal of ABA with these parameters. The assumed memory effect is weakest for the node Vacuolar Acidification; for two timesteps memory the probability of the ON state of this node is $85 \%$ (see Supplementary Text S4). This node is required to be in the ON state for the stabilization of the closureM motif and the turning off of this node contributes to reopening of the stomata. The larger memory duration of three timesteps ensures the persistence of CPK3/21 and MPK9/12 activity. The five timestep memory of Microtubule Depolymerization yields a $97 \%$ probability of its ON state. Overall, these memory durations ensure close to $100 \%$ closure in response to $\mathrm{ABA}$, followed by reopening after the loss of the ABA signal.

\section{Stable Motif Succession Diagram Analysis of the Network With Short-Term Memory}

In the updated model with short term memory, the nodes CPK3/21, MPK9/12, Microtubule Depolymerization and Vacuolar Acidification do not form stable motifs anymore. Indeed, their future state is strictly independent of their own current state and is solely determined by their regulators' current and past states. Since these nodes no longer form motifs, the stable motif succession diagram is modified. However, the short-term memory effect needs to be strong enough to ensure that in the presence of a signal (for example, ABA or external calcium) there is a system trajectory in the succession diagram that leads to the closed stomata attractor. This is possible as ABA or external calcium causes $\mathrm{Ca}^{2+}{ }_{c}$ oscillations, which are sufficient to maintain these four nodes in their ON state if the memory duration is sufficiently large (see Supplementary Text S4 and Figure 8).

\section{ABA OFF Case}

In the absence of ABA, similar to the case described in Section "Stable Motif Succession Diagrams of the Stomatal Closure Model Versions," Model1 has two stable motifs, openM1 and openM2; these motifs are given in Figure 2. The stabilization of either of these two stable motifs causes a fixed OFF state of $\mathrm{Ca}^{2+}{ }_{c}$, which in turn leads to a fixed OFF state of CPK3/21, MPK9/12, Microtubule Depolymerization and Vacuolar Acidification. This leads to an open stomata attractor (see Supplementary Table S4). The presence of only two motifs leads to the possibility of just two trajectories, depicted in Figure 8A.

\section{ABA ON Case}

In the presence of $\mathrm{ABA}$, Model1 has two motifs, one of which is a stable motif comprised of $\mathrm{PA}=1, \operatorname{PLD} \delta=1$ and, $\mathrm{ROS}=1$, and other is the oscillating motif consisting of $\mathrm{Ca}^{2+}{ }_{c}$ and $\mathrm{Ca}^{2+}$ ATPase. This oscillating motif is the same as the one described in the $\mathrm{ABA}=\mathrm{ON}$ case in Section "Stable Motif Succession Diagrams of the Stomatal Closure Model Versions," and as in that case the condition of this motif is satisfied since ABA indirectly activates CaIM. The sustained oscillations of $\mathrm{Ca}^{2+}{ }_{c}$ are sufficient to fix the ON state of CPK3/21 and MPK9/12 if their memory is three or more time steps; they will fix Microtubule Depolymerization and Vacuolar Acidification if their memory is six or more time steps (see Supplementary Text S4). Stabilization of these two motifs (in either order) leads to the closed stomata attractor when the memory duration is large enough (see Figure 8B). If the memory duration is smaller, the system instead converges into 


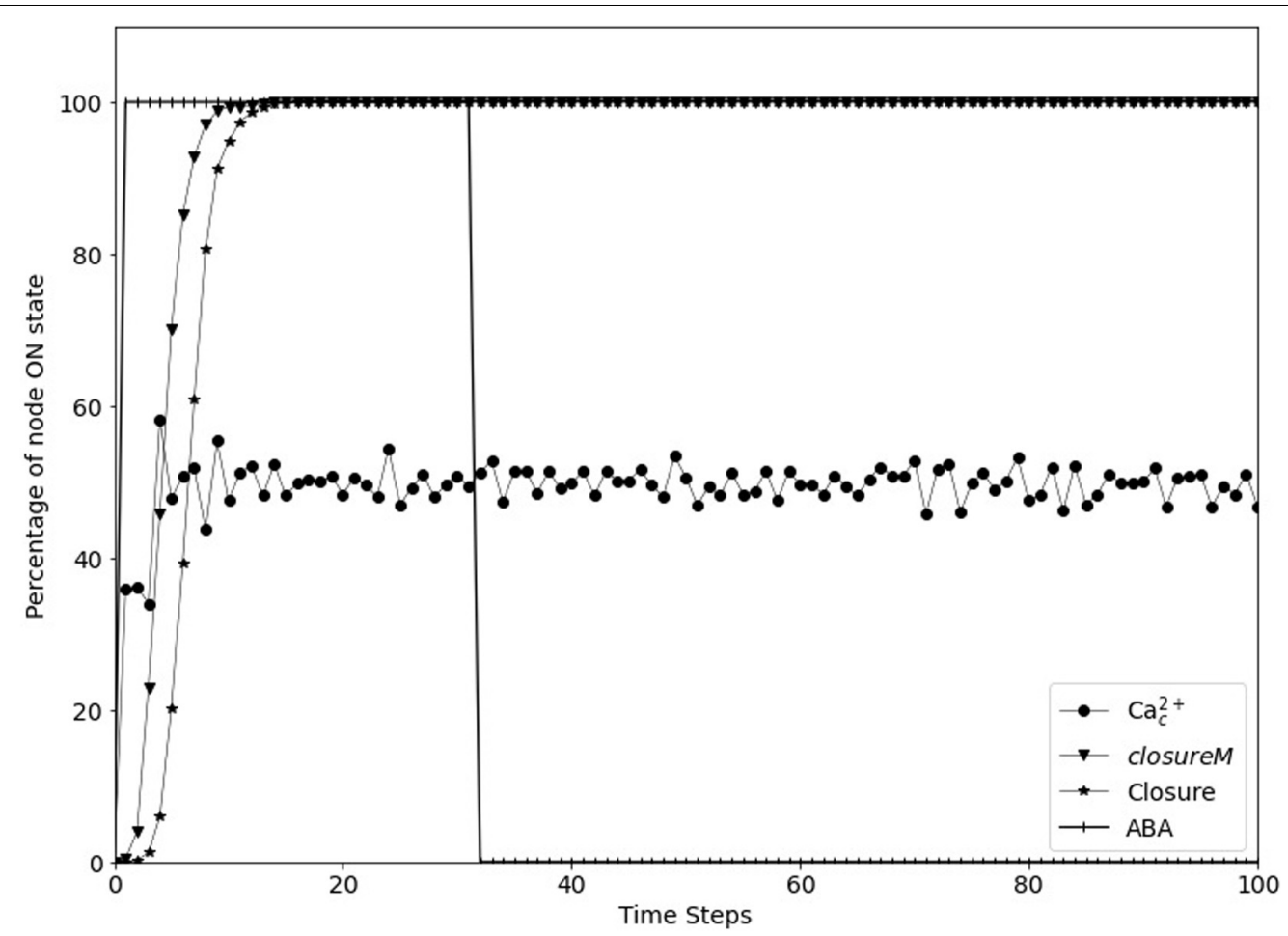

FIGURE 5 | Simulated stomatal closure is maintained after removal of ABA. The state of the node ABA (vertical line symbols) is ON for 30 time-steps and is then OFF. The closureM conditionally stable motif (downward pointing triangles) establishes in less than 15 timesteps and remains stable despite the loss of ABA. The percentage of closure (star symbols) increases to $100 \%$ and stays at this value even after the signal is removed. The circles represent the percentage of the ON state of $\mathrm{Ca}^{2+}{ }_{c}$ which after a fast increase fluctuates around $50 \%$ since $\mathrm{Ca}^{2+}{ }_{c}$ oscillates with approximately equal ON and OFF time periods. The biological expectation is that after the signal is removed the states of ROS, Closure, and $\mathrm{Ca}^{2+}{ }_{c}$ should go to OFF eventually.

an attractor where many nodes, including Closure, oscillate (see Supplementary Table S4). After ABA is removed, the condition for the conditional oscillating motif of $\mathrm{Ca}^{2+}{ }_{c}$ and $\mathrm{Ca}^{2+}$ ATPase is no longer satisfied. Hence, the oscillating motif ceases to exist and the $\mathrm{Ca}^{2+}{ }_{c}$ oscillations decay, which leads to the fixed OFF state of CPK3/21, MPK9/12, Microtubule Depolymerization and Vacuolar Acidification. This in turn leads to an open stomata attractor (see Supplementary Table S4).

\section{Externally Provided $\mathrm{Ca}^{2+}$ as the Signal}

Similar to Section "Stable Motif Succession Diagrams of the Stomatal Closure Model Versions," we considered two methods of simulating external $\mathrm{Ca}^{2+}$. With $\mathrm{Ca}^{2+}{ }_{c}$ fixed in the ON state, the states of CPK3/21, MPK9/12, Microtubule Depolymerization and Vacuolar Acidification are fixed in the ON state too. This leads to activation of the closureM motif, as described in Section "Relaxation to Resting State After Removing the Signal," and hence the network stabilizes in a closed stomata attractor. When external $\mathrm{Ca}^{2+}$ is simulated as fixed ON state of CaIM, the $\mathrm{Ca}^{2+}{ }_{c}-\mathrm{Ca}^{2+}$ ATPase oscillating motif establishes. Indeed, experiments confirm that high external $\mathrm{Ca}^{2+}$ leads to sustained oscillations in $\mathrm{Ca}^{2+}{ }_{c}$ (Jeon et al., 2019). The sustained oscillations of $\mathrm{Ca}^{2+}{ }_{c}$ in the model lead to sustained ON state of CPK3/21, MPK9/12 if their memory is three or more time steps, and to sustained ON state of Microtubule Depolymerization and
Vacuolar Acidification if their memory is six or more time steps. The sustained ON state of Vacuolar Acidification leads to establishment of the closureM motif. The percentage of simulations in which the closureM motif stabilizes is always less than the percentage of $\mathrm{ON}$ state of Vacuolar Acidification and it increases as the memory duration is increased - see Supplementary Figure S7. Once the closureM motif stabilizes, it leads the system to the closed stomata attractor.

The stable motifs and the succession diagram of Model 2 with the short-term memory effect are the same as those of Model1 in the presence of ABA or in the presence of external calcium. Similar to the case described in Section "Stable Motif Succession Diagrams of the Stomatal Closure Model Versions," there are some small differences between the models in the absence of ABA, which are described in Supplementary Text S2.

\section{Recapitulating the Open Stomatal State in the Absence of a Signal}

The model in Maheshwari et al. (2019) yields 20\% closed stomata in simulated wild type guard cells that did not receive any closure signal. We refer to the cumulative percentage of closure (CPC) obtained in the simulation of unstimulated wild type cells as baseline. The biological expectation is that the baseline percentage of closure should be 0 . We determined the baseline percentage of closure in the updated Modell that has short term 
A
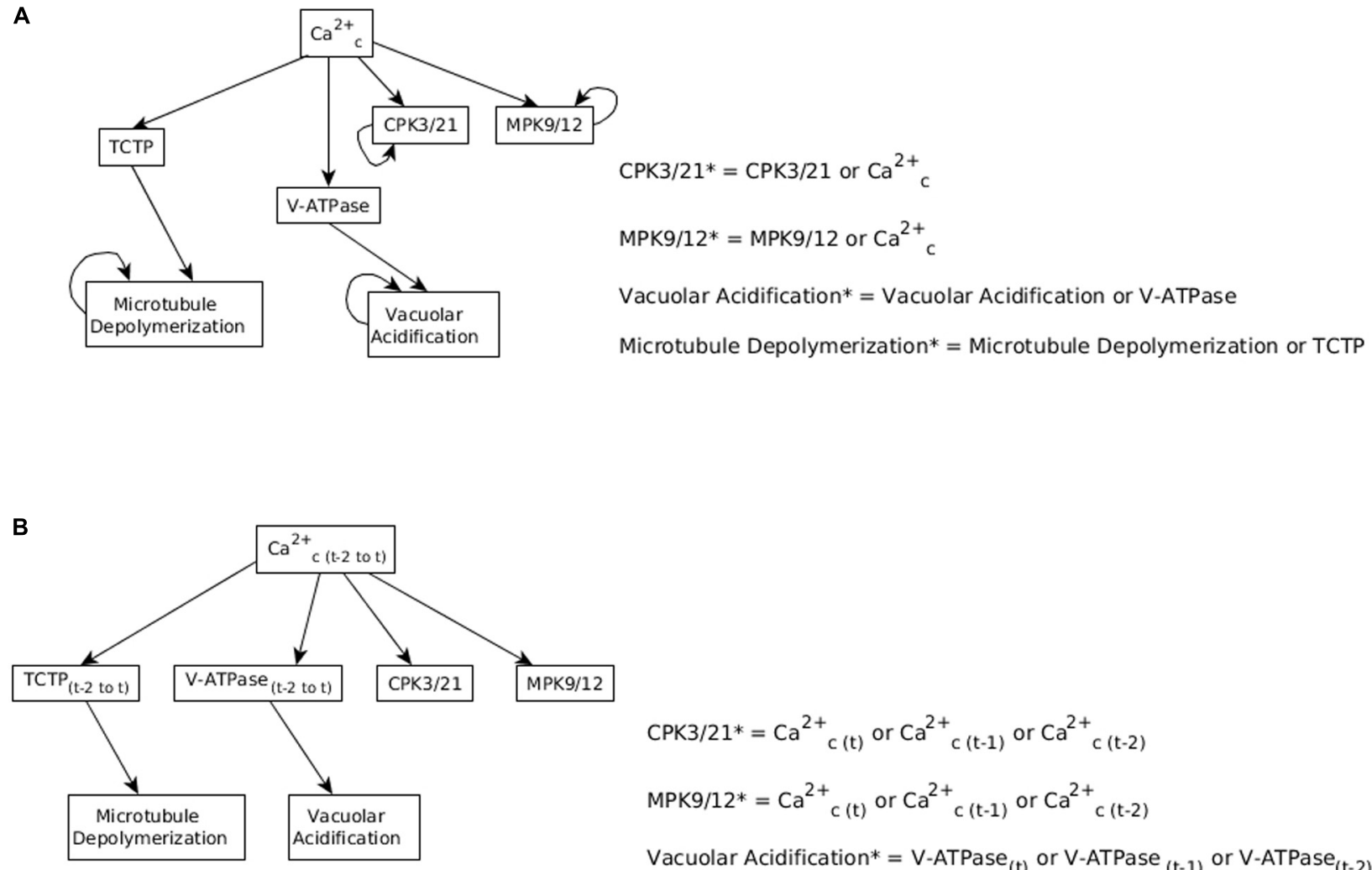

$\mathrm{CPK} 3 / 21^{*}=\mathrm{Ca}^{2+}{ }_{\mathrm{c}(\mathrm{t})}$ or $\mathrm{Ca}^{2+}{ }_{\mathrm{c}(\mathrm{t}-1)}$ or $\mathrm{Ca}^{2+}{ }_{\mathrm{c}(\mathrm{t}-2)}$

MPK9/12* $=\mathrm{Ca}^{2+}{ }_{c(\mathrm{t})}$ or $\mathrm{Ca}^{2+}{ }_{\mathrm{c}(\mathrm{t}-1)}$ or $\mathrm{Ca}^{2+}{ }_{\mathrm{c}(\mathrm{t}-2)}$

Vacuolar Acidification* $=$ V-ATPase $_{(\mathrm{t})}$ or V-ATPase $_{(\mathrm{t}-1)}$ or V-ATPase $_{(\mathrm{t}-2)}$

Microtubule Depolymerization* $=\mathrm{TCTP}_{(\mathrm{t})} \operatorname{or~TCTP}_{(\mathrm{t}-1)} \operatorname{or~TCTP}_{(\mathrm{t}-2)}$

FIGURE 6 | Considering memory of the states of regulator nodes instead of persistent activity. (A) In Maheshwari et al. (2019), the nodes CPK3/21, MPK9/12, Vacuolar Acidification, and Microtubule Depolymerization have a persistence term in their update function to maintain them in a fixed $\mathrm{ON}$ state when $\mathrm{Ca}^{2+}{ }_{C}$ oscillates. (B) Replacing persistent activity with short-term memory. The update function of each of the four nodes combines with an "OR" function the states of their respective regulator $\left(\mathrm{Ca}^{2+}{ }_{c}\right.$, TCTP, or V-ATPase) at the current and previous two time-steps.

memory instead of persistent activity of CPK3/21, MPK9/12, Microtubule Depolymerization, and Vacuolar Acidification. We found that the percentage of closure approaches 0 after a long time; however, there is a non-zero transient level of closure (see Figure 9), and thus a non-zero baseline CPC. While this is a significant improvement compared to the final percentage of $\sim 20 \%$ reported in Maheshwari et al. (2019) and further supports the replacement of persistent activity with short-term memory, this is still not an accurate recapitulation of the biological expectation. Hence, in this section, we explore ways to achieve zero cumulative percentage of closure to better recapitulate the biological expectation.

In Maheshwari et al. (2019), seventeen nodes of the network were initialized randomly because of the lack of experimental evidence regarding their state in open stomata. These nodes are indicated in Supplementary Table S2. We reported in Maheshwari et al. (2019) that the non-zero baseline percentage of stomatal closure can be attributed to the initial condition. When certain nodes are initialized randomly and by chance their initial state is the same as their state in the closed stomata attractor, then the initial state essentially acts as a transient (single timestep) closure signal, which may in certain cases lead to the activation of the closureM motif. As we see in Figure 7, after a closure signal is removed, the percentage of closure, activation of the closureM motif, and $\mathrm{Ca}^{2+}{ }_{c}$ oscillations reduce over time. We observe a similar behavior in Figure 9 where, as a result of the initial condition the percentage of closure, motif stabilization and $\mathrm{Ca}^{2+}{ }_{c}$ oscillations initially increase, and then reduce to less than $5 \%$ after 100 timesteps. The peak of transient percentage of closure, motif stabilization or $\mathrm{Ca}^{2+}{ }_{c}$ oscillations is much lower in Figure 9 than Figure 7 because the probability of activation of the ClosureM motif due to the initial condition is much lower than the probability of activation due to an ABA signal sustained for 30 timesteps. Hence, we hypothesized that the model would yield zero cumulative percentage of closure (CPC) if all of these seventeen nodes were initiated in the state opposite to their state corresponding to closed stomata. This "furthest from closure" initial condition indeed resulted in zero CPC in both Model1 and Model 2 (that is, both when PA inhibits ABI2 and when $\mathrm{Ca}^{2+}{ }_{c}$ is assumed to directly inhibit ABI2).

The "furthest from closure" initial condition assumes a particular state for each of the 17 nodes that were initialized randomly (Maheshwari et al., 2019). Since the pre-stimulus state of none of these nodes was measured experimentally, the validity 


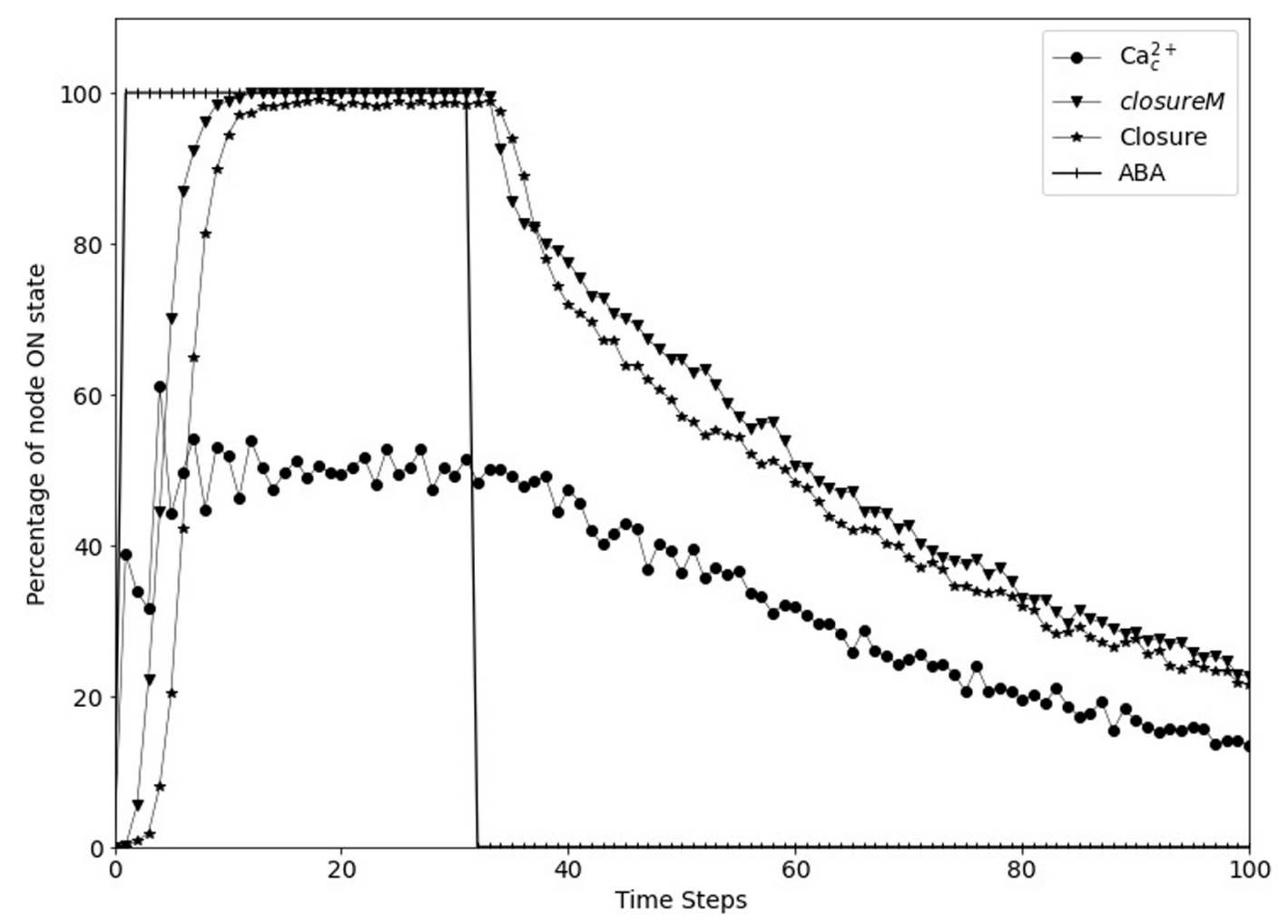

FIGURE 7 | Stomatal re-opening after removal of the signal in the updated Model1. The signal, ABA (vertical lines) is set to fixed ON state for the first 30 time-steps and then set to fixed OFF state for 70 time-steps. The closureM conditionally stable motif (downward triangles) is stabilized within the first 10 time-steps and it slowly destabilizes after ABA is set to the OFF state. The state of the node Closure, depicted by the star symbols, shows a similar behavior i.e., it starts decreasing after the signal is removed. The state of the node $\mathrm{Ca}^{2+}{ }_{c}$, depicted by the circle symbols, is oscillating in the presence of ABA and it slowly transitions to the OFF state after ABA is fixed to OFF state.

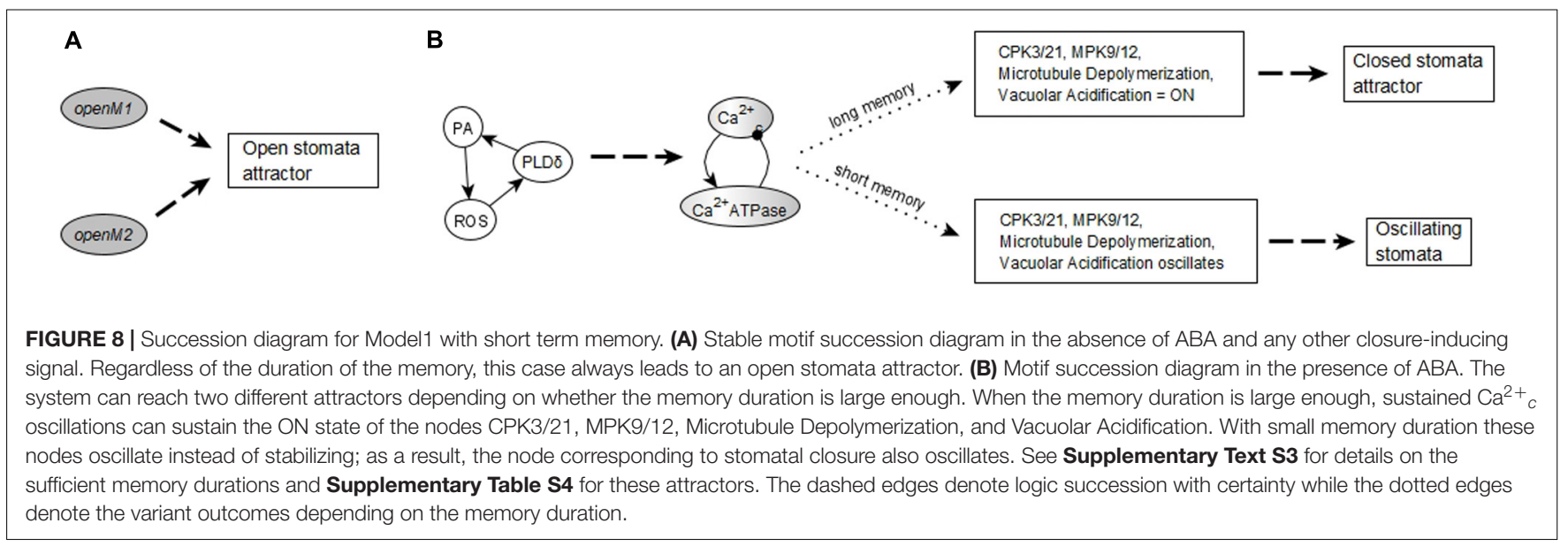

of each of these assumptions is unknown. To reduce the chance of incorrect assumptions, we next explore the possibility of minimizing the number of nodes for which such assumptions are made while still ensuring a zero baseline CPC. We use logical analysis and simulations to find this minimal restriction on initial conditions. In Modell, i.e., the model version where PA inhibits ABI2 through an edge, we find that in order to obtain a baseline CPC of zero, the initial states of 6 of the
17 randomly initialized nodes need to be the opposite of their state corresponding to closed stomata. These six nodes and their corresponding states are $\mathrm{cADPR}=\mathrm{OFF}, \mathrm{GHR} 1=\mathrm{OFF}$, AtRAC $1=$ ON, PLC $=$ OFF, PLD $\delta=$ OFF, and DAG $=$ OFF. All of these nodes affect the closure $M$ conditionally stable motif directly or indirectly. The nodes PLD $\delta$ and DAG are sufficient activators of the node PA, which is an internal driver node of closureM (if Vacuolar Acidification has already stabilized in the ON state). The 


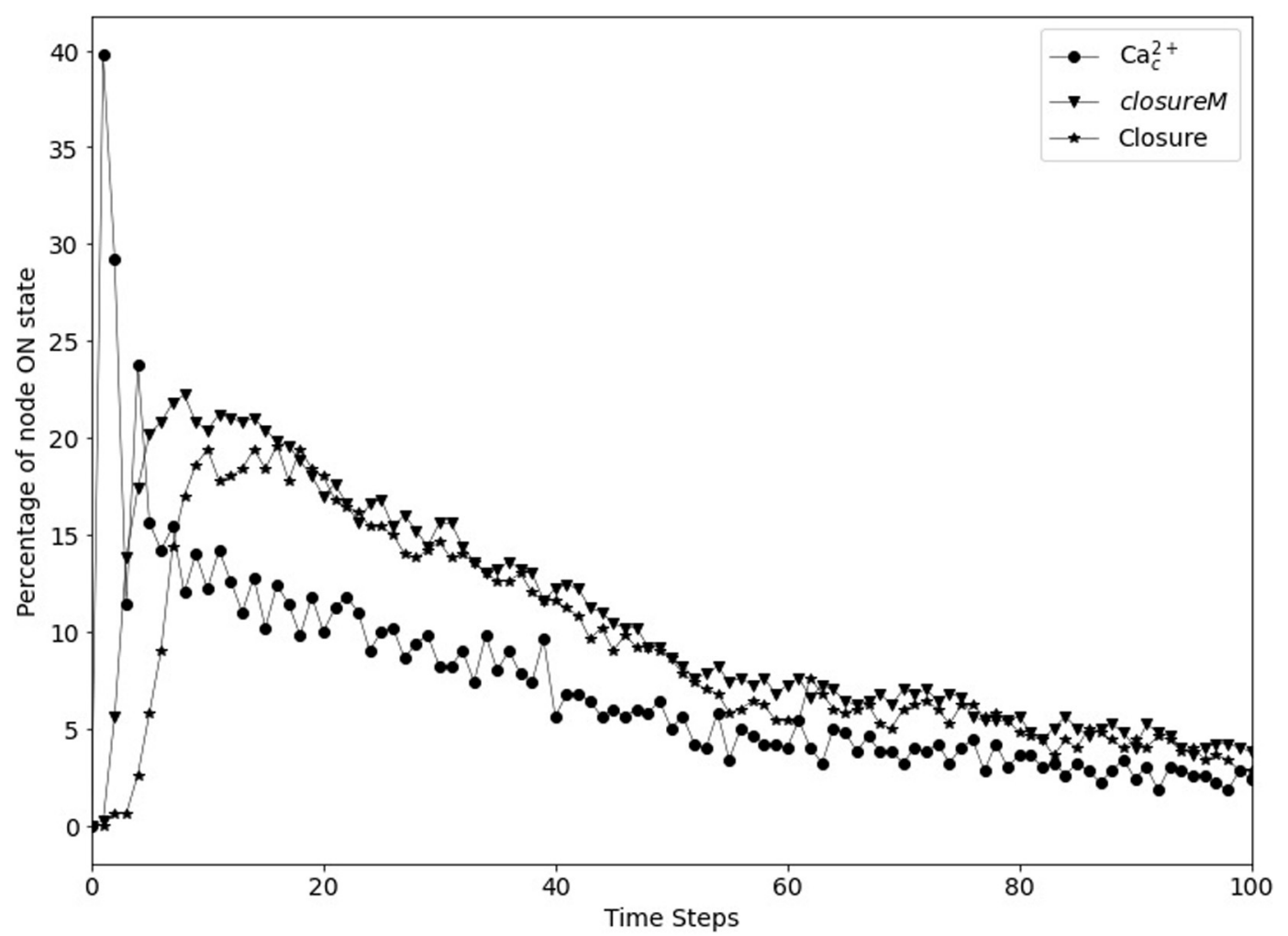

FIGURE 9 | Transient closure observed in Model1 in the absence of ABA when using short-term memory instead of node persistence. Due to the random initial conditions of the 17 nodes, the closure $M$ motif temporarily stabilizes in $\sim 29 \%$ of the simulations (downward triangles) and there is a non-zero level of Ca ${ }^{2+}{ }_{C}$ oscillations, which in turn lead to up to 20\% transient closure. Eventually, the percentage of closure reduces to less than $5 \%$.

remaining four nodes, i.e., cADPR, GHR1, AtRAC1, and PLC, promote either $\mathrm{Ca}^{2+}$ influx through the membrane (CaIM) or $\mathrm{Ca}^{2+}$ release from intracellular stores (CIS), hence promoting $\mathrm{Ca}^{2+}{ }_{c}$ oscillations - see the purple nodes in Figure 10. $\mathrm{Ca}^{2+}{ }_{c}$ is an external driver of closureM (Maheshwari et al., 2019), as it can induce the $\mathrm{ON}$ state of the Vacuolar Acidification node (the condition of the closure $M$ motif) and induce the $\mathrm{ON}$ state of $\mathrm{PA}$, the $\mathrm{ON}$ state of $\mathrm{pH}_{c}$ and the OFF state of ABI2. Since these 3 nodes form a 3 -node driver of this conditionally stable motif, the $\mathrm{ON}$ state of $\mathrm{Ca}^{2+}{ }_{c}$ leads to stabilization of closureM (Maheshwari et al., 2019). Sustained oscillations of $\mathrm{Ca}^{2+}{ }_{c}$ can also lead to the stabilization of the closureM motif if the relationship between the $\mathrm{Ca}^{2+}{ }_{c}=\mathrm{ON}$ period and the shortterm memory of Vacuolar Acidification is such that the Vacuolar Acidification $=\mathrm{ON}$ condition is satisfied (see Maheshwari et al., 2019, Supplementary Text S3, and Supplementary Figure S7). Hence if any of these four nodes are initialized in their state corresponding to stomatal closure, there is a chance of indirectly stabilizing the closure $M$ motif and to transiently driving the system to closure.

In Model2, i.e., the model version where the inhibition of ABI2 happens through a direct edge from $\mathrm{Ca}^{2+}{ }_{c}$, we found that a baseline CPC of zero is achieved when the initial state of just four of the 17 nodes is specified and the remaining 13 are initialized randomly. These four nodes are shown in purple background in Figure 10; their corresponding states are cADPR $=$ OFF, GHR $1=$ OFF, AtRAC $1=$ ON and PLC $=$ OFF. These four nodes are a subset of the six nodes we identify in the case of Model1; specifically, they are the nodes that can promote $\mathrm{Ca}^{2+}{ }_{c}$ oscillations. Since in Model $2 \mathrm{Ca}^{2+}{ }_{c}$ directly inhibits ABI2, the initial conditions that promote $\mathrm{Ca}^{2+}{ }_{c}$ have a slightly higher likelihood of leading to closure. In Model2 PA is not an internal driver of the closureM motif (Maheshwari et al., 2019), thus the initial state of nodes DAG or PLD $\delta$ does not play a role in promoting the stabilization of this motif and hence promoting closure. In summary, Model 2 requires fewer restrictions than Model1 to avoid the activation, in the absence of ABA, of the closure $M$ conditionally stable motif.

We evaluated the agreement of these model versions, sampling their respective sets of initial conditions that correspond to zero baseline closure percentage, with the extant experimental evidence for the effects of constitutive activation of nodes (e.g., whether constitutive activation of a node induces a significant decrease in stomatal aperture, see Section "Evaluation of Consistency Between Simulation and Experiment"). The model-indicated effects of constitutive activation of single nodes fall into three categories: close to baseline response (which in this case means a CPC of zero), slightly increased response, and significantly increased response (which in this case leads to a final closure percentage of $100 \%)$. In Model1, i.e., the model 


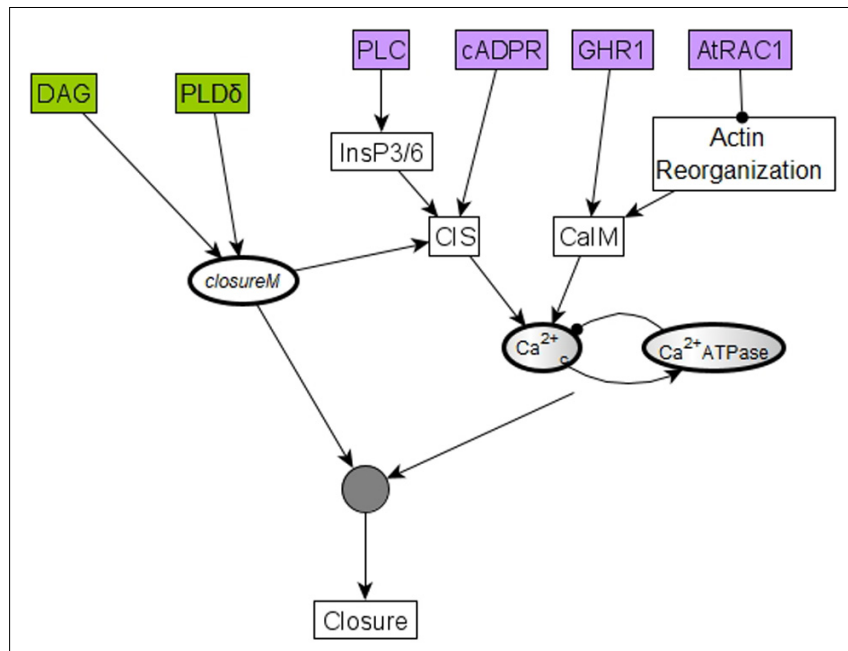

FIGURE 10 | The initial condition of six nodes can lead to temporary closure by helping establish $\mathrm{Ca}^{2+}{ }_{\mathrm{C}}$ oscillations and the closureM conditionally stable motif. Four of these six nodes, CADPR, GHR1, AtRAC1, and PLC,

represented in purple, regulate one of the two processes (CIS or CalM) that yield $\mathrm{Ca}^{2+}{ }_{\mathrm{c}}$ elevation. For example, the two-edge path between AtRAC1 and CalM indicates that AtRAC1 inhibits the reorganization of the actin cytoskeleton that otherwise would contribute to $\mathrm{Ca}^{2+}$ influx (CalM). $\mathrm{Ca}^{2+}{ }_{\mathrm{C}}$ elevation, in turn, has some probability of causing $\mathrm{Ca}^{2+}{ }_{c}$ oscillations due to the negative feedback loop formed by $\mathrm{Ca}^{2+}{ }_{C}$ and $\mathrm{Ca}^{2+}{ }_{c}$ ATPase. These oscillations have a potential to drive the network to the closed stomata attractor. The remaining two nodes, PLD $\delta$ and DAG, represented in green, affect the closure $M$ motif, indicated by the edges that start from PLD $\delta$ and DAG, respectively, and end in the node that stands for the closureM motif. Both nodes are direct regulators of PA, which is an internal driver of the motif, thus their activity has a chance of locking in the closureM motif. The stabilization of the closureM motif is sufficient for sustained $\mathrm{Ca}^{2+}{ }_{c}$ oscillations, indicated by the path mediated by CIS. The cumulative effect of sustained $\mathrm{Ca}^{2+}{ }_{c}$ oscillations and of the closure $\mathrm{M}$ motif leads to stomatal closure (see Section "Stable Motif Succession Diagrams of the Stomatal Closure Model Versions"). Hence, initiating any of these six nodes in their states corresponding to stomatal closure leads to a non-zero percentage of stomatal closure, at least transiently.

version where PA directly inhibits $\mathrm{ABI}$, the simulations agree with experimental observations in 14 instances and they disagree in 3 instances (see Table $\mathbf{1}$ and Supplementary Table S5). The instances of agreement include six cases in which the model and experiments agree in observing closure (decreased stomatal aperture) in case of constitutive activation of the corresponding node and 8 cases in which neither the model nor experiments observe closure. The instances of agreement include the constitutive activation of CaIM, which happens in experiments where $\mathrm{Ca}^{2+}$ is provided externally (see last entry of Table 1). The experimental observation of $\mathrm{Ca}^{2+}{ }_{c}$ oscillations, as well as stomatal closure, in response to the presence of external $\mathrm{Ca}^{2+}$ (Jeon et al., 2019) also supports the model prediction that the sustained $\mathrm{Ca}^{2+}{ }_{c}$ oscillations are sufficient for stomatal closure.

Conversely, Model1 yields a zero percentage of closure in case of constitutive activity of $\mathrm{S} 1 \mathrm{P}$ and $\mathrm{pH}_{c}$ (as neither S1P nor $\mathrm{pH}_{c}$ can independently stabilize the closureM motif) while a decreased aperture was observed experimentally. In case of
PLD $\alpha$ constitutive activity Model 1 yields slightly higher than baseline closure (as PLD $\alpha$ leads to PA production, which is an internal driver of the closureM motif) while experiments did not observe a statistically significant decrease in stomatal aperture. In the "furthest from closure" initial condition (when nodes are initialized in the state opposite their state corresponding to stomatal closure) there are 13 cases of agreement and four cases of disagreement. The 13 cases of agreement include four cases of significantly increased closure and nine cases of close to baseline closure. In addition to $\mathrm{S} 1 \mathrm{P}$ and $\mathrm{pH}_{c}$, there is also disagreement for external supply of PA and NO, where the model yields close to baseline response but a higher than baseline degree of closure was observed experimentally. Similar to the case of S1P and $\mathrm{pH}_{c}$, $\mathrm{PA}$ and NO cannot independently stabilize the closureM motif. They can however stabilize the motif in a fraction of cases when initial conditions are favorable.

In Model2, i.e., the model version where $\mathrm{Ca}^{2+}{ }_{c}$ directly inhibits ABI2, we found the same instances of agreement (13) and disagreement (4) as for the "furthest from closure" initial conditions of Model1 (see Supplementary Table S6). The reason for the cases of disagreement between experimental results and simulation results remains the same as for Model1: none of S1P, $\mathrm{NO}, \mathrm{PA}$, or $\mathrm{pH}_{c}$ can stabilize the closureM motif. Each of them can only activate the motif as part of a two- or three-node driver set (e.g., the two-node driver set of PA and Vacuolar Acidification and the three-node driver set of S1P, Vacuolar Acidification, and PLD $\delta$ ). The same categories of responses to node constitutive activations, and thus the same cases of disagreement, were found for the "furthest from closure" initial condition and for the least restrictive initial condition that yields a baseline percentage of closure of zero.

We next sought to determine whether initiating one or more nodes of the previously restricted sets, i.e., (cADPR, GHR1, AtRAC1, PLC) or (cADPR, GHR1, AtRAC1, PLC, PLD $\delta, D A G)$, respectively, in a random state would still maintain near-zero baseline closure while increasing the percentage of closure when $\mathrm{S} 1 \mathrm{P}, \mathrm{NO}, \mathrm{PA}$ or $\mathrm{pH}_{c}$ is constitutively activated. We indeed found this to be the case. A few slightly less restricted initial conditions in Model1 lead to a non-zero but small value of transient baseline closure (see Supplementary Table S7). These cases show improved agreement with experiments regarding node constitutive activation compared to the least restricted initial condition summarized in column 2 of Supplementary Table S6. Specifically, the constitutive activation of $\mathrm{pH}_{c}$ is now in the slightly increased category, in agreement with experimental results, while its categorization in Supplementary Table S6 as yielding close to baseline response disagreed with experiments. The node $\mathrm{pH}_{c}$ alone is not sufficient to be a driver of the closureM motif; however, it can contribute to 2-node or 3-node drivers when combined with the initial activity of DAG or PLD $\delta$. When the system starts in an initial state that includes DAG or active $\operatorname{PLD} \delta$ there is a non-zero probability of the closureM motif stabilizing and leading to the closed stomata attractor.

In Model2, we found initial conditions where the simulations of node constitutive activations include fewer cases of disagreement with experimental results than the initial conditions that ensure a CPC of zero, but these initial conditions 


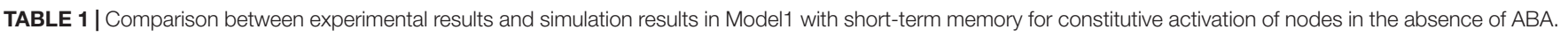

\begin{tabular}{|c|c|c|c|}
\hline $\begin{array}{l}\text { Node that is } \\
\text { constitutively active }\end{array}$ & $\begin{array}{l}\text { In silico response when using the initial } \\
\text { condition furthest from closure }\end{array}$ & $\begin{array}{l}\text { In silico response when using the } \\
\text { least restricted initial condition }\end{array}$ & Experimentally observed response \\
\hline TCTP & Close to baseline & Close to baseline & Close to baseline (Kim et al., 2012) \\
\hline ROP11 & Close to baseline & Close to baseline & Close to baseline (Li et al., 2012) \\
\hline $\begin{array}{l}\text { Microtubule } \\
\text { Depolymerization }\end{array}$ & Close to baseline & Close to baseline & Close to baseline (Jiang et al., 2014) \\
\hline PLD $\alpha$ & Close to baseline & Slightly increased & Close to baseline (Mishra et al., 2006) \\
\hline PA & Close to baseline & Slightly increased & Increased (Jacob et al., 1999) \\
\hline $\mathrm{NO}$ & Close to baseline & Slightly increased & Increased (Desikan et al., 2002) \\
\hline S1P & Close to baseline & Close to baseline & Increased (Ng et al., 2001; Coursol et al., 2003) \\
\hline AtRAC1 & Close to baseline & Close to baseline & Close to baseline (Lemichez et al., 2001) \\
\hline $\mathrm{H}^{+}$ATPase & Close to baseline & Close to baseline & Close to baseline (Wang et al., 2014) \\
\hline PP2CA & Close to baseline & Close to baseline & Close to baseline (Kuhn et al., 2006) \\
\hline $\mathrm{ABl1}$ & Close to baseline & Close to baseline & Close to baseline (Allen et al., 1999) \\
\hline $\mathrm{ABI} 2$ & Close to baseline & Close to baseline & Close to baseline (Allen et al., 1999) \\
\hline cADPR & Significantly increased & Significantly increased & Increased (Joudoi et al., 2013) \\
\hline InsP3/6 & Significantly increased & Significantly increased & Increased (Gilroy et al., 1990) \\
\hline ROS & Significantly increased & Significantly increased & Increased (Zhang et al., 2001; Kwak et al., 2003) \\
\hline CalM & Significantly increased & Significantly increased & Increased (Jeon et al., 2019) \\
\hline
\end{tabular}

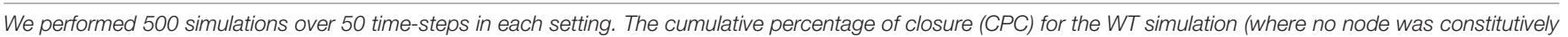

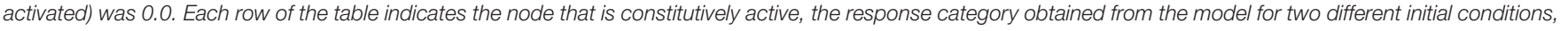

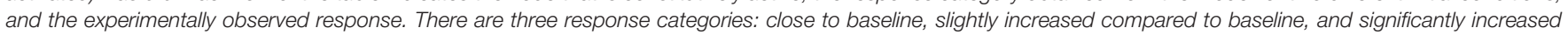

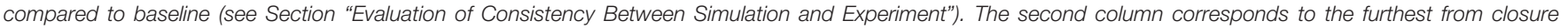

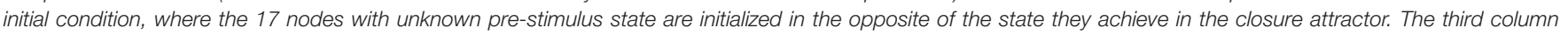

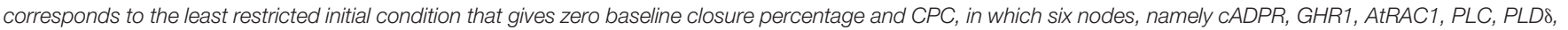

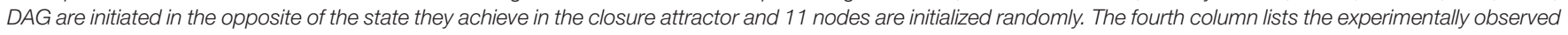

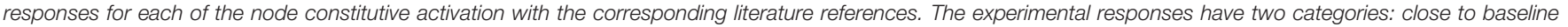

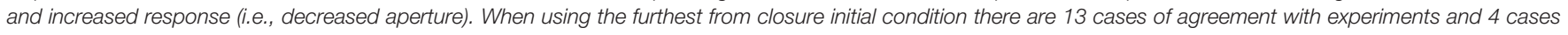

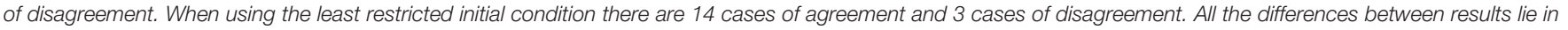
the constitutive activation of PLD $\alpha, P A, S 1 P$ and NO. Supplementary Table S6 indicates the CPC values of each case.

also result in a CPC that is significantly higher than zero. For example, random initialization of PLC yields a higher than baseline closure in case of constitutive activation of each of S1P, $\mathrm{PA}$, and $\mathrm{pH}_{c}$, with the only remaining disagreement being NO. The trade-off for this regained agreement of the simulated node constitutive activation with experiments is an increase in the baseline percentage of closure and CPC: the peak percentage of closure is $3 \%$ and the CPC is 1.91 . In conclusion, it is not possible to simultaneously ensure a zero baseline percentage of closure and recapitulate closure in response to constitutive activity of $\mathrm{S} 1 \mathrm{P}$ or $\mathrm{pH}_{c}$ given our current knowledge of the biological resting/open stomata state. The node initializations that help achieve better agreement with experimental data on node constitutive activations also increase the baseline percentage of closure.

\section{DISCUSSION}

We present ways to improve the Boolean model of ABA-induced stomatal closure by Maheshwari et al. (2019) to recapitulate biological expectations regarding stomata remaining in an open state in the absence of closure signals, or relaxing back to the open stomata state following the loss of closure signals. We find that modifying the assumed persistent activity of four nodes to a short-term memory effect helps recapitulate re-opening of the stomata after the closure signal is removed. The implementation of such short-term memory still yields transient closure in the absence of any closure signal; thus we also identify different combinations of initial conditions that minimize this transient closure. We find that the percentage of stomatal closure is sensitive to the initial states of certain nodes. This highlights the significance of these internal nodes and the importance of experimentally determining the resting states (or open stomata states) of these nodes.

Motivated by the incorporation of timing in Boolean modeling in Thakar et al. (2007), we replaced the assumed persistent activity of four nodes, i.e., CPK3/21, MPK9/12, Microtubule Depolymerization, and Vacuolar Acidification, with a function that considers the cumulative effect of the current and the past states of their regulator. We show that this short-term memory effect of an oscillating regulator, for example, $\mathrm{Ca}^{2+}{ }_{c}$, helps these four nodes maintain the persistent activity necessary to ensure stomatal closure in response to closure-inducing signals. This assumption brings the model closer to biological reality by exhibiting reopening of the stomata after the closureinducing signal is removed. We perform a systematic study of the effect of varying memory durations on the extent of stomatal closure and the rate of reopening upon removal of the signal. Our analysis of the increase in the percentage of $\mathrm{ON}$ state of a node for larger memory duration can be extended to other patterns of oscillations of the regulator node and hence it will be useful in various other Boolean models of biological networks. 
Our analysis demonstrates that motif succession diagrams provide a powerful means to present and understand the system trajectories, highlighting the points of no return in the system's dynamics and identifying the various attractors the system can lead to. In this work, we present the motif succession diagram of the stomatal closure network, which integrates and summarizes previous research on this network (Li et al., 2006; Albert et al., 2017; Maheshwari et al., 2019). This succession diagram highlights the key role of oscillating motifs, drawing attention to the significance of oscillations in this network. This advances our understanding of node oscillations in the context of a biological system modeled as a Boolean network. Seeking motivation from recent work (Deritei et al., 2019), we also identify conditionally stable and conditional oscillating motifs and differentiate them from their condition-free counterparts.

The motif succession diagram is also an effective measure of the consequences of short-term versus long-term memory. Comparing Figure 8A with Figure 3, we can see that shortterm memory eliminates the trajectories that would yield a closure attractor in the absence of $\mathrm{ABA}$ and reduces the variability of the open stomatal attractors. Another way to illustrate the qualitative difference between short-term and long-term memory is through a bifurcation diagram (Tyson and Novak, 2020), which indicates the steady state value(s) of the node Closure for different values of ABA. As described in Supplementary Text S5, an on-off-on sequence of ABA yields an irreversible switch in the status of closure in case of persistent activity of CPK3/21, MPK9/12, Microtubule Depolymerization and Vacuolar Acidification, while shortterm memory of the same nodes yields reversible closure. Comparing Figure $\mathbf{8 B}$ with Figure $\mathbf{4}$, we can see that shortterm memory leads to an attractor in which the Closure node oscillates in the presence of ABA. The fact that oscillating stomatal apertures have not been observed experimentally in response to $\mathrm{ABA}$ suggests that the biological persistence of vacuolar acidification, microtubule depolymerization and of the activity of $\mathrm{CPK} 3 / 21$ and MPK9/12 is essential to the control of oscillations. This also highlights the importance of characterizing and quantifying the biological mechanisms underlying the persistence of these nodes (see Supplementary Text S1).

This work and our previous research on stable motifs (Zañudo and Albert, 2013; Steinway et al., 2014; Albert et al., 2017; Zanudo and Albert, 2015; Maheshwari et al., 2019; Rozum and Albert, 2018; Gan and Albert, 2018; Deritei et al., 2019) contributes to the broader field of investigation that connects positive feedback loops, multistability, cell fates and phenotypes (Thomas and Ari, 1990; Huang, 2007; Hari et al., 2020). Specifically, single or intersecting positive feedback loops form stable motifs or conditionally stable motifs. Mutually exclusive stable motifs determine distinct attractors (distinct phenotypes). For example, in the model studied here the openM1 stable motif and closureM conditionally stable motif are mutually exclusive (see Figure 3A). The stabilization of one of two mutually exclusive stable motifs at the expense of the other represents a bifurcation in the system's trajectory toward a specific phenotype. The specific example in this work is the possibility of a trajectory toward closed stomata in the absence of ABA. Such a bifurcation can be viewed as a cellular decision point. Our work suggests a mechanism of cellular plasticity (phenotype switching): destabilization of the conditionally stable motif that underlies the phenotype by deactivating its condition. As seen in previous work (Deritei et al., 2019), stable motifs that are condition-free within the context of one model can become conditionally stable motifs in a broader model that encompasses the original model but includes more regulators and processes.

Our analysis of initial conditions of 17 nodes with uncertain states found that it is not possible to simultaneously have a baseline cumulative percentage of closure of 0 and also recapitulate the experimentally observed closure for constitutive activation of $\mathrm{pH}_{c}, \mathrm{PA}, \mathrm{NO}$ or S1P (Jacob et al., 1999; Ng et al., 2001; Desikan et al., 2002; Coursol et al., 2003; Mishra et al., 2006; Gonugunta et al., 2008). This discrepancy suggests that the actual guard cell resting/open state does not correspond to the state farthest from the state associated with stomatal closure. In order to ensure optimal response over a range of conditions, certain nodes have to already be "primed" (be in their state associated with stomatal closure) prior to receiving the closure stimulus. The effectiveness of such priming has been documented in the case of $\mathrm{Ca}^{2+}{ }_{c}$ stimulus: when guard cells were pre-exposed to $\mathrm{ABA}$ or $\mathrm{CO}_{2}$, elevated $\mathrm{Ca}^{2+}{ }_{c}$ strongly activated $\mathrm{S}$-type anion channels by shifting their $\mathrm{Ca}^{2+}{ }_{c}$ sensitivity to lower levels (Israelsson et al., 2006; Hubbard et al., 2012; Laanemets et al., 2013). This suggestion is also in accordance with previous experimental observations that the cellular changes underlying stomatal closure (e.g., induced by ABA) are not simply the reverse of the processes underlying stomatal opening (e.g., induced by light) (Assmann, 1993; Wang et al., 2001; Yin et al., 2013; He et al., 2018). Such "flexible" nodes may provide important portals for regulation by other stimuli to which multisensory guard cells also respond, including not only $\mathrm{CO}_{2}$ concentrations but also blue and red light, humidity, and pathogens (Sun et al., 2014; Murata et al., 2015; Assmann and Jegla, 2016; Engineer et al., 2016).

\section{DATA AVAILABILITY STATEMENT}

The original and updated network versions analyzed for this study can be found in the following github repository: https: //github.com/parulm/Stomata_PP2Cs.

\section{AUTHOR CONTRIBUTIONS}

PM, SA, and RA designed the research and methodology, and wrote the manuscript. PM and RA performed the analyses. All authors contributed to the article and approved the submitted version. 


\section{FUNDING}

This work was supported by NSF grant MCB-1715826 to SA and RA and NSF grant IIS-1814405 to RA. The funders had no role in study design, data collection and analysis, decision to publish, or preparation of the manuscript.

\section{ACKNOWLEDGMENTS}

We thank Jorge G. T. Zanudo, Xiao Gan, and Jordan Rozum for their helpful discussions.

\section{SUPPLEMENTARY MATERIAL}

The Supplementary Material for this article can be found online at: https://www.frontiersin.org/articles/10.3389/fphys. 2020.00927/full\#supplementary-material

\section{REFERENCES}

Ache, P., Becker, D., Ivashikina, N., Dietrich, P., Roelfsema, M. R. G., and Hedrich, R. (2000). GORK, a delayed outward rectifier expressed in guard Cells of Arabidopsis thaliana, Is a $\mathrm{K}^{+}$-Selective, $\mathrm{K}^{+}$-Sensing ion channel. Febs Lett. 486, 93-98. doi: 10.1016/s0014-5793(00)02248-1

Albert, I., Thakar, J., Li, S., Zhang, R., and Albert, R. (2008). Boolean network simulations for life scientists. Source Code Biol. Med. 3:16.

Albert, R., Acharya, B. R., Jeon, B. W., Zañudo, J. G., Zhu, M., Osman, K., et al. (2017). A new discrete dynamic model of ABA-induced stomatal closure predicts key feedback loops. PLoS Biol. 15:e2003451. doi: 10.1371/journal.pbio. 2003451

Albert, R., DasGupta, B., Dondi, R., Kachalo, S., Sontag, E., Zelikovsky, A., et al. (2007). A novel method for signal transduction network inference from indirect experimental evidence. J. Comput. Biol. 14, 927-949. doi: 10.1089/cmb.2007. 0015

Allen, G. J., Kuchitsu, K., Chu, S. P., Murata, Y., and Schroeder, J. I. (1999). Arabidopsis ABI1-1 and ABI2-1 phosphatase mutations reduce abscisic acidinduced cytoplasmic calcium rises in guard cells. Plant Cell 11, 1785-1798. doi: $10.1105 /$ tpc.11.9.1785

Assmann, S. M. (1993). Signal transduction in guard cells. Annu. Rev. Cell Biol. 9, 345-375. doi: 10.1146/annurev.cb.09.110193.002021

Assmann, S. M., and Jegla, T. (2016). Guard Cell Sensory systems: recent insights on stomatal responses to light, abscisic acid, and $\mathrm{CO}_{2}$. Curr. Opin. Plant Biol. 33, 157-167. doi: 10.1016/j.pbi.2016.07.003

Coursol, S., Fan, L.-M., Le Stunff, H., Spiegel, S., Gilroy, S., and Assmann, S. M. (2003). Sphingolipid signalling in arabidopsis guard cells involves heterotrimeric G proteins. Nature 423, 651-654. doi: 10.1038/nature 01643

Cummins, W. R., Kende, H., and Raschke, K. (1971). Specificity and reversibility of the rapid stomatal response to abscisic acid. Planta 99, 347-351. doi: 10.1007/ bf00385826

Deritei, D., Rozum, J., Regan, E. R., and Albert, R. (2019). A feedback loop of conditionally stable circuits drives the cell cycle from checkpoint to checkpoint. Sci. Rep. 9, 1-19.

Desikan, R., Griffiths, R., Hancock, J., and Neill, S. (2002). A new role for an old enzyme: nitrate reductase-mediated nitric oxide generation is required for abscisic acid-induced stomatal closure in Arabidopsis thaliana. Proc. Natl. Acad. Sci. U.S.A. 99, 16314-16318. doi: 10.1073/pnas.252461999

Engineer, C. B., Hashimoto-Sugimoto, M., Negi, J., Israelsson-Nordström, M., Azoulay-Shemer, T., Rappel, W.-J., et al. (2016). $\mathrm{CO}_{2}$ sensing and $\mathrm{CO}_{2}$ regulation of stomatal conductance: advances and open questions. Trends Plant Sci. 21, 16-30. doi: 10.1016/j.tplants.2015.08.014

Gan, X., and Albert, R. (2016). Analysis of a dynamic model of guard cell signaling reveals the stability of signal propagation. BMC Syst. Biol. 10:78.
TABLE S1 | The full names corresponding to the abbreviated node names in the 49-node ABA induced closure network.

TABLE S2 | Initial conditions of all nodes in the model, reproduced from the 49-node model.

TABLE S3 | The Boolean update function of each node in Model1.

TABLE S4 | Attractors of Model1 and Model2.

TABLE S5 | Attractors of Model1 with short-term memory.

TABLE S6 | Simulation results from Model1 and Model2 with short-term memory for constitutive activation of nodes in the absence of ABA for initial conditions that ensure the absence of baseline closure.

TABLE S7 | Simulation results for constitutive activation of each node in the absence of ABA in Model1 with short term memory for initial conditions that result in near zero baseline percentage of closure.

TEXT S1 | Biological reasoning for the assumed slow decay of the activity of four network elements.

TEXT S2 | Succession diagram for Model2.

Gan, X., and Albert, R. (2018). General method to find the attractors of discrete dynamic models of biological systems. Phys. Rev. E 97:042308.

Gilroy, S., Read, N., and Trewavas, A. J. (1990). Elevation of cytoplasmic calcium by caged calcium or caged inositol trisphosphate initiates stomatal closure. Nature 346, 769-771. doi: 10.1038/346769a0

Gonugunta, V. K., Srivastava, N., Puli, M. R., and Raghavendra, A. S. (2008). Nitric oxide production occurs after cytosolic alkalinization during stomatal closure induced by abscisic acid. Plant Cell Environ. 31, 1717-1724. doi: 10.1111/j. 1365-3040.2008.01872.x

Grondin, A., Rodrigues, O., Verdoucq, L., Merlot, S., Leonhardt, N., and Maurel, C. (2015). Aquaporins contribute to ABA-triggered stomatal closure through OST1-mediated phosphorylation. Plant Cell 27, 1945-1954. doi: 10.1105/tpc. 15.00421

Hari, K., Sabuwala, B., Subramani, B. V., La Porta, C. A., Zapperi, S., FontClos, F., et al. (2020). Identifying inhibitors of epithelial-mesenchymal plasticity using a network topology-based approach. NPJ Syst. Biol. Appl. $6,1-12$.

He, J., Zhang, R.-X., Peng, K., Tagliavia, C., Li, S., Xue, S., et al. (2018). The $\mathrm{BIG}$ protein distinguishes the process of $\mathrm{CO}_{2}$-induced stomatal closure from the inhibition of stomatal opening by $\mathrm{CO}_{2}$. New Phytol. 218, 232-241. doi: 10.1111/nph.14957

Hosy, E., Vavasseur, A., Mouline, K., Dreyer, I., Gaymard, F., Porée, F., et al. (2003). The Arabidopsis Outward $\mathrm{K}^{+}$channel GORK is involved in regulation of stomatal movements and plant transpiration. Proc. Natl. Acad. Sci. U.S.A. 100, 5549-5554. doi: 10.1073/pnas.0733970100

Huang, S. (2007). Cell Fates as Attractors-Stability and Flexibility of Cellular Phenotype: Endothelial Biomedicine, 1 Edn. New York, NY: Cambridge University Press, 1761-1779.

Hubbard, K. E., Siegel, R. S., Valerio, G., Brandt, B., and Schroeder, J. I. (2012). Abscisic acid and $\mathrm{CO}_{2}$ signalling via calcium sensitivity priming in guard cells, new CDPK mutant phenotypes and a method for improved resolution of stomatal stimulus-response analyses. Ann. Bot. 109, 5-17. doi: 10.1093/aob/ mcr 252

Israelsson, M., Siegel, R. S., Young, J., Hashimoto, M., Iba, K., and Schroeder, J. I. (2006). Guard cell $\mathrm{ABA}$ and $\mathrm{CO}_{2}$ signaling network updates and $\mathrm{Ca}^{2+}$ sensor priming hypothesis. Curr. Opin. Plant Biol. 9, 654-663. doi: 10.1016/j.pbi.2006. 09.006

Jacob, T., Ritchie, S., Assmann, S. M., and Gilroy, S. (1999). Abscisic acid signal transduction in guard cells is mediated by phospholipase D activity. Proc. Natl. Acad. Sci. 96, 12192-12197. doi: 10.1073/pnas.96.21.12192

Jeon, B. W., Acharya, B. R., and Assmann, S. M. (2019). The Arabidopsis heterotrimeric G-protein $\beta$ subunit, AGB 1, is required for guard cell calcium sensing and calcium-induced calcium release. Plant J. 99, 231-244. 
Jiang, Y., Wu, K., Lin, F., Qu, Y., Liu, X., and Zhang, Q. (2014). Phosphatidic acid integrates calcium signaling and microtubule dynamics into regulating abainduced stomatal closure in Arabidopsis. Planta 239, 565-575. doi: 10.1007/ s00425-013-1999-5

Joudoi, T., Shichiri, Y., Kamizono, N., Akaike, T., Sawa, T., Yoshitake, J., et al. (2013). Nitrated cyclic GMP modulates guard cell signaling in Arabidopsis. Plant Cell 25, 558-571. doi: 10.1105/tpc.112.105049

Kim, Y.-M., Han, Y.-J., Hwang, O.-J., Lee, S.-S., Shin, A.-Y., Kim, S. Y., et al. (2012). Overexpression of arabidopsis translationally controlled tumor protein gene AtTCTP enhances drought tolerance with rapid aba-induced stomatal closure. Mol. Cells 33, 617-626. doi: 10.1007/s10059-012-0080-8

Kollist, H., Nuhkat, M., and Roelfsema, M. R. G. (2014). Closing Gaps: linking elements that control stomatal movement. New Phytol. 203, 44-62. doi: 10. $1111 /$ nph. 12832

Kuhn, J. M., Boisson-Dernier, A., Dizon, M. B., Maktabi, M. H., and Schroeder, J. I. (2006). The protein phosphatase AtPP2CA negatively regulates abscisic acid signal transduction in arabidopsis, and effects of Abh1 on AtPP2CA MRNA. Plant Physiol. 140, 127-139. doi: 10.1104/pp.105.070318

Kwak, J. M., Mori, I. C., Pei, Z.-M., Leonhardt, N., Torres, M. A., Dangl, J. L., et al. (2003). NADPH oxidase AtrbohD and AtrbohF genes function in ROSdependent ABA signaling in Arabidopsis. EMBO J. 22, 2623-2633. doi: 10.1093/ emboj/cdg277

Laanemets, K., Brandt, B., Li, J., Merilo, E., Wang, Y.-F., Keshwani, M. M., et al. (2013). Calcium-dependent and-independent stomatal signaling network and compensatory feedback control of stomatal opening via $\mathrm{Ca}^{2+}$ sensitivity priming. Plant Physiol. 163, 504-513. doi: 10.1104/pp.113.22 0343

Lemichez, E., Wu, Y., Sanchez, J.-P., Mettouchi, A., Mathur, J., and Chua, N.-H. (2001). Inactivation of AtRacl by abscisic acid is essential for stomatal closure. Genes Dev. 15, 1808-1816. doi: 10.1101/gad.900401

Li, S., Assmann, S. M., and Albert, R. (2006). Predicting essential components of signal transduction networks: a dynamic model of guard cell abscisic acid signaling. PLoS Biol. 4:e312. doi: 10.1371/journal.pbio.00 40312

Li, Z., Kang, J., Sui, N., and Liu, D. (2012). ROP11 GTPase is a negative regulator of multiple aba responses in Arabidopsis. J. Integrat. Plant Biol. 54, 169-179. doi: 10.1111/j.1744-7909.2012.01100.x

Ma, Y., Szostkiewicz, I., Korte, A., Moes, D., Yang, Y., Christmann, A., et al. (2009). Regulators of PP2C phosphatase activity function as abscisic acid sensors. Science 324, 1064-1068.

Maheshwari, P., and Albert, R. (2017). A framework to find the logic backbone of a biological network. BMC Syst. Biol. 11:122.

Maheshwari, P., Du, H., Sheen, J., Assmann, S. M., and Albert, R. (2019). Modeldriven discovery of calcium-related protein-phosphatase inhibition in plant guard cell signaling. PLoS Comput. Biol. 15:e1007429. doi: 10.1371/journal.pcbi. 1007429

Mishra, G., Zhang, W., Deng, F., Zhao, J., and Wang, X. (2006). A bifurcating pathway directs abscisic acid effects on stomatal closure and opening in Arabidopsis. Science 312, 264-266. doi: 10.1126/science.1123769

Murata, Y., Mori, I. C., and Munemasa, S. (2015). Diverse stomatal signaling and the signal integration mechanism. Ann. Rev. Plant Biol. 66, 369-392. doi: 10.1146/annurev-arplant-043014-114707

Ng, C. K.-Y., Carr, K., McAinsh, M. R., Powell, B., and Hetherington, A. M. (2001). Drought-induced guard cell signal transduction involves sphingosine1-phosphate. Nature 410, 596-599. doi: 10.1038/35069092

Roelfsema, M. R. G., and Hedrich, R. (2005). In the light of stomatal opening: new insights into 'the watergate.'. New Phytol. 167, 665-691. doi: 10.1111/j.14698137.2005.01460.x
Rozum, J. C., and Albert, R. (2018). Self-sustaining positive feedback loops in discrete and continuous systems. J. Theoret. Biol. 459, 36-44. doi: 10.1016/j. jtbi.2018.09.017

Sierla, M., Waszczak, C., Vahisalu, T., and Kangasjärvi, J. (2016). Reactive oxygen species in the regulation of stomatal movements. Plant Physiol. 171, 1569-1580. doi: $10.1104 / p p .16 .00328$

Staxén, I., Pical, C., Montgomery, L. T., Gray, J. E., Hetherington, A. M., and McAinsh, M. R. (1999). Abscisic acid induces oscillations in guard-cell cytosolic free calcium that involve phosphoinositide-specific Phospholipase C. Proc. Natl. Acad. Sci. U.S.A. 96, 1779-1784. doi: 10.1073/pnas.96.4.1779

Steinway, S. N., Zañudo, J. G., Ding, W., Rountree, C. B., Feith, D. J., Loughran, T. P., et al. (2014). Network modeling of TGF $\beta$ signaling in hepatocellular carcinoma epithelial-to-mesenchymal transition reveals joint sonic hedgehog and wnt pathway activation. Cancer Res. 74, 5963-5977. doi: 10.1158/00085472.can-14-0225

Sun, Z., Jin, X., Albert, R., and Assmann, S. M. (2014). Multi-level modeling of light-induced stomatal opening offers new insights into its regulation by drought. PLoS Comput. Biol. 10:e1003930. doi: 10.1371/journal.pcbi.1003930

Thakar, J., Pilione, M., Kirimanjeswara, G., Harvill, E. T., and Albert, R. (2007). Modeling systems-level regulation of host immune responses. PLoS Comput. Biol. 3:e109. doi: 10.1371/journal.pcbi.0030109

Thomas, R., and Ari, R. D. (1990). Biological Feedback. Boca Raton, FL: CRC press. Tyson, J. J., and Novak, B. (2020). A dynamical paradigm for molecular cell biology. Trends Cell Biol. 504-515. doi: 10.1016/j.tcb.2020.04.002

Wang, R.-S., Saadatpour, A., and Albert, R. (2012). Boolean modeling in systems biology: an overview of methodology and applications. Phys. Biol. 9:055001. doi: 10.1088/1478-3975/9/5/055001

Wang, X.-Q., Ullah, H., Jones, A. M., and Assmann, S. M. (2001). G protein regulation of ion channels and abscisic acid signaling in Arabidopsis guard cells. Science 292, 2070-2072. doi: 10.1126/science. 1059046

Wang, Y., Noguchi, K., Ono, N., Inoue, S., Terashima, I., and Kinoshita, T. (2014). Overexpression of plasma membrane $\mathrm{H}^{+}$-ATPase in guard cells promotes lightinduced stomatal opening and enhances plant growth. Proc. Natl. Acad. Sci. U.S.A. 111, 533-538. doi: 10.1073/pnas.1305438111

Yin, Y., Adachi, Y., Ye, W., Hayashi, M., Nakamura, Y., Kinoshita, T., et al. (2013). Difference in abscisic acid perception mechanisms between closure induction and opening inhibition of stomata. Plant Physiol. 163, 600-610. doi: 10.1104/ pp.113.223826

Zañudo, J. G., and Albert, R. (2013). An effective network reduction approach to find the dynamical repertoire of discrete dynamic networks. Chaos 23:025111. doi: $10.1063 / 1.4809777$

Zanudo, J. G., and Albert, R. (2015). Cell fate reprogramming by control of intracellular network dynamics. PLoS Comput. Biol. 11:e1004193. doi: 10.1371/ journal.pcbi.1004193

Zhang, X., Zhang, L., Dong, F., Gao, J., Galbraith, D. W., and Song, C.-P. (2001). Hydrogen peroxide is involved in abscisic acid-induced stomatal closure in vicia faba. Plant Physiol. 126, 1438-1448. doi: 10.1104/pp.126.4.1438

Conflict of Interest: The authors declare that the research was conducted in the absence of any commercial or financial relationships that could be construed as a potential conflict of interest.

Copyright $\odot 2020$ Maheshwari, Assmann and Albert. This is an open-access article distributed under the terms of the Creative Commons Attribution License (CC BY). The use, distribution or reproduction in other forums is permitted, provided the original author(s) and the copyright owner(s) are credited and that the original publication in this journal is cited, in accordance with accepted academic practice. No use, distribution or reproduction is permitted which does not comply with these terms. 